\title{
Just how long can you live in a black hole and what can be done about it?
}

\author{
Gregory A. Burnett \\ Department of Physics, University of Florida, Gainesville, Florida 32611
}

(19 April 1995)

\begin{abstract}
We study the problem of how long a journey within a black hole can last. Based on our observations, we make two conjectures. First, for observers that have entered a black hole from an asymptotic region, we conjecture that the length of their journey within is bounded by a multiple of the future asymptotic "size" of the black hole, provided the spacetime is globally hyperbolic and satisfies the dominant-energy and non-negative-pressures conditions. Second, for spacetimes with $\mathbb{R}^{3}$ Cauchy surfaces (or an appropriate generalization thereof) and satisfying the dominant energy and non-negative-pressures conditions, we conjecture that the length of a journey anywhere within a black hole is again bounded, although here the bound requires a knowledge of the initial data for the gravitational field on a Cauchy surface. We prove these conjectures in the spherically symmetric case. We also prove that there is an upper bound on the lifetimes of observers lying "deep within" a black hole, provided the spacetime satisfies the timelike-convergence condition and possesses a maximal Cauchy surface. Further, we investigate whether one can increase the lifetime of an observer that has entered a black hole, e.g., by throwing additional matter into the hole. Lastly, in an appendix, we prove that the surface area $A$ of the event horizon of a black hole in a spherically symmetric spacetime with ADM mass $M_{\mathrm{ADM}}$ is always bounded by $A \leq 16 \pi M_{\mathrm{ADM}}^{2}$, provided that future null infinity is complete and the spacetime is globally hyperbolic and satisfies the dominant-energy condition.
\end{abstract}

04.70.Bw, 04.20.Dw, 04.20.Cv 


\section{INTRODUCTION AND SUMMARY}

Over the past forty years, black-hole physics has become quite a mature field [1,2]. Driven by a desire to understand their fundamental properties and potential importance to astrophysics, the study of black holes has produced a number of outstanding results. For example, Hawking has proven that black holes never bifurcate and that the areas of black-hole event horizons never decrease to the future [3, 1 . Further, through the efforts of a number of researchers, the black hole "no-hair" theorems [4, [] have established that stationary electrovacuum black holes are far simpler objects than were once imagined [2,6]. In all of these studies, the interiors of black holes have been entirely ignored, as well they should as these regions neither affect nor are observable from the asymptotic region of the spacetime (by their very definition). So, while a great deal has been learned about black-hole exteriors, comparatively little is known about black-hole interiors.

Are there any features that black-hole interiors share? There are at least two results in this direction. First, Penrose has shown that if a spacetime contains a future trapped surface then, provided the cosmic censorship hypothesis holds 17 10, 4 , it must lie within the black-hole region and that such a spacetime must be singular in the sense that it cannot be future null geodesically complete [11,3, $[$ ]. While a truly amazing and important theorem in the history of black-hole physics, it tells us very little about the extent to which the spacetime is singular. Are just a few, most, or all of the geodesics in the interior region incomplete? How long does it take for such a singularity to develop? Second, a number of people using a variety of methods (see [12] and the references therein), have examined whether the Cauchy horizons that occur in the interiors of the Kerr-Newman black holes will be present in more "realistic" spacetimes. In particular, Poisson and Israel have argued that these horizons are destroyed (generically) due to an unbounded "inflation" of the mass function $m$ on what was the Cauchy horizon inside the black hole [12,13]. Although arguments have been given that indicate that these results are quite general, a proof remains to be found [14, 15].

Here, in the effort to explore the features of black-hole interiors, we examine a single issue: How long can the journey of an observer in a black hole last? Must it be finite? If so, can an upper bound on the length of such a journey be given in terms of some characteristic of the black hole? It turns out that there are two forms of this problem that are best considered separately. First, we restrict ourselves with observers that enter a black hole from the asymptotic region. That is, we are restricting our attention to the portion of a black-hole region lying to the future of past null infinity $\mathcal{J}^{-}$. Second, we drop this restriction and look at the entire black-hole region. However, we shall see that this generalization does come at a small price. A restriction on the Cauchy surface topology is necessary (while none appears necessary for the first case) along with a weaker bound on the lifetimes of observers within the black-hole region.

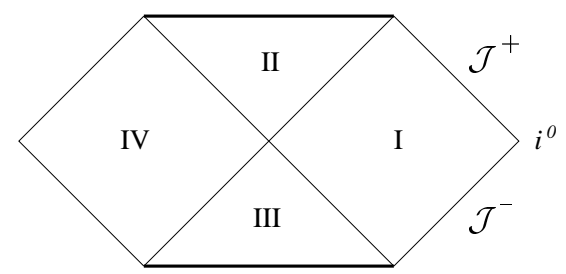

FIG. 1. A spacetime diagram representing a maximally extended Schwarzschild spacetime of positive mass $M$. The black-hole region (according to $\mathcal{J}^{+}$), is all of regions II and IV. An observer beginning a journey in the asymptotic region $\langle\langle\mathcal{J}\rangle\rangle$ (region I) and entering the black hole must enter region II. A journey in this region cannot last more than a time $\pi M$ (and this maximum is attained for journeys perpendicular to the surfaces of spatial homogeneity).

Consider first the case of an observer entering a black hole from an asymptotic region [16]

$$
\langle\langle\mathcal{J}\rangle\rangle=J^{-}\left(\mathcal{J}^{+}\right) \cap J^{+}\left(\mathcal{J}^{-}\right),
$$

also known as the domain of outer communications [6. In the maximally extended Schwarzschild spacetime, this is region II depicted in Fig. 11. A partial answer to our problem can obtained from the work of Friedman, Schleich, and Witt which can be stated as: In an asymptotically flat spacetime satisfying the null-convergence condition [18] with distinct null infinities $\mathcal{J}_{i}$, the sets $J^{-}\left(\mathcal{J}_{i}^{+}\right) \cap J^{+}\left(\mathcal{J}_{j}^{-}\right)$are empty for $i \neq j$ [17]. Therefore, fixing one null infinity $\mathcal{J}_{0}$ it is impossible for an observer to leave the asymptotic region $\left\langle\left\langle\mathcal{J}_{0}\right\rangle\right\rangle$ by entering a black hole and then live indefinitely by escaping to another asymptotic region. The maximally extended Schwarzschild spacetime depicted in Fig. 1 exemplifies this impossibility. Although this result is very encouraging, it does not eliminate other possibilities for an observer existing indefinitely within a black hole. Based on the general considerations presented in Sec. II and its successful proof in the spherically symmetric case (theorem 1 below), we conjecture that this result can indeed be strengthened.

Conjecture 1. Fix a globally hyperbolic asymptotically flat spacetime satisfying the dominant-energy and non-negative-pressures conditions [18] with a non-empty black-hole region. Fix a connected component $\mathcal{E}_{0}$ of the event horizon and denote the supremum of its area as measured over the set of all of its spatial cross-sections by $A_{f}$. Then, there exists a constant $k$ (independent of which spacetime and event horizon is chosen) such that the lifetime of any observer within the black hole is bounded above by $k \sqrt{A_{f} / 16 \pi}$ provided that the black hole is entered by crossing $\mathcal{E}_{0}$ in the future of $\mathcal{J}^{-}$.

Recall that the irreducible mass associated with a connected component of black-hole's event horizon at a given 
"time" is obtained by calculating its surface area $A$ on a spatial cross-section and setting [4]

$$
M_{\mathrm{irr}}=\sqrt{A / 16 \pi}
$$

The quantity is "irreducible" as it is non-decreasing to future, which follows from the fact that the area $A$ of the event horizon is non-decreasing to the future [3, 4. So, with this definition, conjecture 1 bounds the lifetime of observers within a black hole by a multiple of the supremum of the irreducible mass calculated on the black-hole's event horizon (provided the black hole is entered from the asymptotic region).

To explore the features of conjecture 1, consider a black-hole spacetime associated with two distant stars that each collapses forming black holes. Should these two black holes never collide with one another, the event horizon will then have two connected components and conjecture 1 asserts that the lifetime of an observer that enters one of the black holes (to the future of $\mathcal{J}^{-}$) is bounded by a quantity computable from the geometry of the event horizon of the black hole entered. However, should the two black holes collide and coalesce into a single black hole, the event horizon will then have a single connected component. In this case, the bound given by conjecture 1 is insensitive as to whether the black hole was entered before or after coalescence and to which black hole was entered. So, the bound is the same for observers entering a small black hole and a large black hole as long as the two eventually coalesce. This is probably a gross overestimate in the case of an observer entering the smaller black hole before coalescence and probably can be refined.

Next, suppose we slightly modify conjecture 1 by having it place a bound on the lifetime of observers in the portion of the black hole lying to the future of $\mathcal{J}^{-}$. Fix any two points $p$ and $q$ in this region with $q \in J^{+}(p)$ and fix any causal curve $\nu$ from $\mathcal{J}^{-}$to $p$. Then, as $\nu$ must intersect the event horizon somewhere, conjecture 1 gives an upper bound on the lengths of all causal curves from $p$ to $q$ (that is independent of $p$ of $q$ ), thereby bounding the lifetimes of all observers in this region. However, suppose that the black hole is sufficiently complicated that its boundary (part if not all of the total event horizon) is not connected. For example, we could imagine a spacetime containing a wormhole with its mouths sufficiently separated and moving in such a way that the event horizons enclosing each mouth never coalesce. Then, if $p$ can be reached from $\mathcal{J}^{-}$by two causal curves that intersect different horizons (i.e., thread different mouths), we would have a choice of which component to use in establishing an upper bound to the lifetimes of observers therein. Can we use the smaller of the two, or must we always use the larger? Or should we use a bound based on total area of the event horizons associated with our region? We leave this problem open for investigation.

In conjecture 1, we have limited ourselves to observers entering a black hole from an asymptotic region. Might the bound given by conjecture 1 hold for all observers within a given black hole? Clearly not, if we do not put some restriction on the Cauchy surface topology as the maximally extended Schwarzschild spacetime provides a simple counterexample. Here, the problem is that there as an entire asymptotic region within the black hole. (See Fig. 1.) Even if we restrict our consideration to spacetimes with Cauchy surfaces having the topology of a compact orientable connected three-manifold $C$ minus a point (so that there are no other asymptotic regions to escape to) then, as is demonstrated in Sec. III, if we do not further restrict $C$, asymptotically flat black-hole spacetimes can be constructed with "compact internal regions" and yet admitting infinitely long timelike curves in the black-hole region. Furthermore, as is demonstrated in Sec. II, even if we restrict ourselves to spacetimes with $\mathbb{R}^{3}$ Cauchy surfaces, there is no $k$ for which the bound given by conjecture 1 will hold. However, here the only problem appears to be that the bound is not quite appropriate. The idea is that the bound in conjecture 1 is constructed from the geometry of the event horizon and therefore can be calculated without a knowledge of the interior of the black hole. For more general observers, a knowledge of the interior geometry (at least on a Cauchy surface) is necessary in obtaining a bound on the lifetimes of observers therein. Based on these considerations and its successful proof in the spherically symmetric case (theorem 2 below), we are led to the following conjecture.

Conjecture 2. Fix a globally hyperbolic asymptotically flat spacetime satisfying the dominant-energy and nonnegative-pressures conditions [18]. Then, provided the Cauchy surfaces are diffeomorphic to $\mathbb{R}^{3}$, there is a number $k$ (independent of which spacetime is chosen) and a quantity $M_{\Sigma}$ computable from the initial data on a Cauchy surface $\Sigma$, such that the lifetimes of all observers within the black-hole region is bounded above by $k M_{\Sigma}$.

In this conjecture, $M_{\Sigma}$ is intended to represent the least upper bound to a quasilocal mass function (associating a number with each given spacelike two-sphere in a spacetime) over the set of all two-spheres in $\Sigma$. As we have not specified what quasilocal mass function is to be used (see however Ref. [19] and the references therein), this portion of the conjecture is imprecise and we leave the task of making it precise as part of the problem. However, when we specialize to the spherically symmetric spacetimes, we expect this mass to be $\operatorname{simply} \sup _{\Sigma}(m)$, where $m$ is the mass function constructible in any spherically symmetric spacetime as given by Eq. (4.2) in Sec. IV A.

For simplicity, we have limited the Cauchy surface topology in conjecture 2 to $\mathbb{R}^{3}$. In Sec. III, we generalize this conjecture by broadening the class of spatial topologies. Furthermore, a theorem is presented which states (roughly) that the lengths of causal curves in the portion of the black hole not reachable from the asymptotic region are bounded provided the spacetime admits a maximal Cauchy surface. Although this is far from a proof of conjecture 2, it is encouraging.

As the calculation of $A_{f}$ in conjecture 1 requires a 
knowledge of the geometry of spacetime in the far future (and therefore can be quite difficult to calculate in practice) one might worry that $A_{f}$ could be arbitrarily large (or even infinite) thereby making the bound given by conjecture 1 rather weak. However, from an analysis of the spherically symmetric case (see theorem A1 in Appendix A), we expect the three masses $M_{\mathrm{irr}}, M_{\mathrm{ADM}}$, and $M_{\Sigma}$ to be related according to the following inequalities

$$
M_{\mathrm{irr}} \leq M_{\mathrm{ADM}} \leq M_{\Sigma}
$$

Whether the second inequality actually holds cannot be investigated until $M_{\Sigma}$ is given a precise definition. However, since the total area $A_{T}$ of the black hole at a given "time" is the sum of the areas associated with each connected component, the first inequality always holds provided that $A_{T} \leq 16 \pi M_{\mathrm{ADM}}^{2}$. (This inequality is similar to, though distinct from, the Gibbons-Penrose isoperimetric inequality for initial data sets in general relativity which has its inspiration from this inequality 2024.) That such an inequality should hold can be argued as follows. Consider this inequality in an asymptotically flat electrovacuum spacetime whose event horizon is connected (i.e., so there is only one black hole at late times). As the area of the event horizon is non-decreasing to the future, this inequality is most difficult to satisfy at late times. However, it is expected that the black hole will eventually settle-down and approach a stationary state which, by the "no-hair" theorems, must be one of the Kerr-Newman spacetimes where it is known that the inequality does indeed hold. (As far as the author is aware, whether this inequality always holds is unresolved. Theorem A1 in Appendix A proves it does hold for the spherically symmetric spacetime satisfying the dominantenergy condition.) Therefore, should the first inequality in Eq. (1.3) indeed hold, then the bound given by conjecture 1 will always be finite. Furthermore, independent of this inequality, an alternate version of conjecture 1 can be obtained by replacing the bound by $k M_{\mathrm{ADM}}$. Should the irreducible mass always be bounded by the ADM mass (as expected), then conjecture 1 would imply this alternate version.

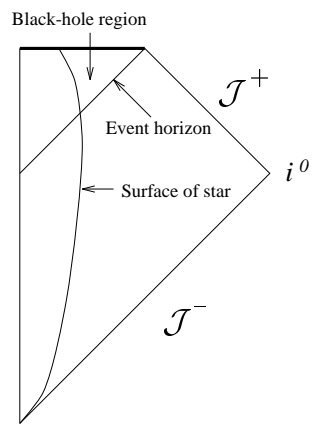

FIG. 2. A spacetime diagram representing the collapse of a star forming a black hole. Notice that, in this simple case, the event horizon can be used as a Cauchy surface for the black-hole region.
Conjectures 1 and 2 are similar to the conjecture that closed universes possessing $S^{3}$ or $S^{1} \times S^{2}$ Cauchy surfaces (and obeying the dominant-energy and non-negativepressures conditions) must have finite lifetimes [25 28], which is a slight variant of the closed-universe recollapse conjecture [29 32]. In fact, the similarity is more than superficial. The idea is that the requirement that the Cauchy surfaces be compact in the closed-universe finite lifetime conjecture can probably be replaced by the more general requirement that the geometry on Cauchy surfaces be bounded in an appropriate sense. In the spherically symmetric case we can make this precise. If we replace the requirement that the Cauchy surfaces be compact with bounds on $r$ and $m$, we can again establish a finite lifetime for the spacetime. (Actually, if this is done, the final bound on the lifetime may be slightly different from that stated in Ref. [28].) With this in mind, consider the collapse of a star leading to the formation of a black hole as depicted in Fig. 2. The black-hole region $B$ of this spacetime is globally hyperbolic with its boundary $\mathcal{E}=\partial B$ (the event horizon of the black hole) acting as a Cauchy surface for this region. Although $\mathcal{E}$ is non-compact it does enjoy the property that the geometry thereon is essentially bounded. In particular, the area of the black hole (as measured on the event horizon) is bounded by its future asymptotic value. Therefore, it would seem that proofs of these two conjectures would be very similar and that one could be obtained from the other with only slight modifications being required. Indeed, a proof of conjectures 1 and 2 for the spherically symmetric case can be obtained using the techniques developed in the course of the proof that spherically symmetric closed universes have finite lifetimes. In fact, we prove somewhat more.

Theorem 1. Fix a globally hyperbolic asymptotically flat spherically symmetric spacetime satisfying the dominant-energy and non-negative-pressures conditions [18] and possessing a Cauchy surface that is geodesically complete. Then, the lifetime of an observer in the black hole is bounded above by $20 \sup _{\mathcal{E}}\left(M_{\mathrm{irr}}\right)$ provided either: (a) the black hole is entered from the asymptotic region of the spacetime; or (b) the spacetime possesses a spherically symmetric Cauchy surface with $\nabla^{a} r$ everywhere outgoing; or (c) the observer lies to the future of the component of a Cauchy surface connected to $i^{0}$ on which $\nabla^{a} r$ is outgoing.

Note that in the spherically symmetric case, on a sphere of symmetry of area $A=4 \pi r^{2}, M_{\text {irr }}$ is simply $r / 2$. As shown by theorem A1 in Appendix A, provided the dominant-energy condition holds, for spheres on the event horizon $M_{\text {irr }} \leq M_{\mathrm{ADM}}$ (i.e., $r / 2$ on the event horizon is always bounded above by $M_{\mathrm{ADM}}$ ). Therefore, the bound in theorem 1 can always be replaced by $20 M_{\mathrm{ADM}}$ thereby given a bound that is calculable from the initial data on a Cauchy surface. Further, provided that the event horizon is complete in the sense given by theorem A2 in Appendix A, then $M_{\mathrm{irr}}=r / 2$ and $m$ have the same future asymptotic limits on the event horizon. 
Therefore, in this case, the bound in theorem 1 is equivalent to 20 times the future asymptotic value of the mass $m$ on the event horizon.

Each part of theorem 1 has its own strengths and weaknesses. Part (a) of this theorem is best used when we know that the observer is entering the black hole from the asymptotic region. However, suppose we are given the location of an observer on a (spherically symmetric) initial data surface $\Sigma$ (a Cauchy surface) and that he enters the black hole. Do we need to find the rest of the spacetime from the initial data on $\Sigma$ to find out whether the observer was indeed in the asymptotic region? No. If $\nabla^{a} r$ is outgoing on all of $\Sigma$, then by (b) the length of his (and indeed all observers) journey in the black hole is bounded by a multiple of the future asymptotic irreducible mass of the black hole. However, suppose that $\nabla^{a} r$ is not outgoing on all of $\Sigma$ (i.e., $\Sigma$ contains a future or past outer trapped surface). Then, provided our observer begins his journey on the portion of $\Sigma$ connected to $i^{0}$ on which $\nabla^{a} r$ is outgoing, then, by (c), his lifetime within the black hole is still bounded by a multiple of the future asymptotic irreducible mass of the black hole.

In theorem 1, we have demanded that the spacetime admit a Cauchy surface that is geodesically complete. This is automatically satisfied by asymptotically flat Cauchy surfaces with $\mathbb{R}^{3}$ topology, but not may not be satisfied by those with $\mathbb{R} \times S^{2}$ topology. We make this requirement as it guarantees that $m$ will be everywhere non-negative, which is needed for the method of proof used herein (see lemma 1). It is likely, though not for certain, that the proof can be improved upon, allowing us to lift the requirement that a Cauchy surface be complete.

The next theorem gives an upper bound on the lifetimes of observers within the black-hole region of a spherically symmetric spacetime that makes no requirements on how the black hole was entered nor on how $\nabla^{a} r$ behaves on a Cauchy surface. Note that the price for this is that a restriction on the Cauchy topology is necessary and that the bound need not be as tight as that given by theorem 1.

Theorem 2. Fix an asymptotically flat spherically symmetric spacetime that possesses $\mathbb{R}^{3}$ Cauchy surfaces and that satisfies the dominant-energy and non-negativepressures conditions [18]. Then, for any Cauchy surface $\Sigma$, the lifetime of an observer in the black-hole region $B$ with event horizon $\mathcal{E}=\partial B$ is bounded above by

$$
20 \max \left(\sup _{\mathcal{E}}\left(M_{\mathrm{irr}}\right), \max _{(\Sigma \cap B) \cup P}(m)\right),
$$

where $P$ is the subset of $\Sigma$ on which $\nabla^{a} r$ is past-directed timelike, past-directed null, or zero.

To understand the features of the bound given by theorem 2, consider the black hole formed by the collapse of a spherical star from a state that is benign in the sense that on a spherically symmetric Cauchy surface $\Sigma$ there are no future or past outer trapped surfaces (so in particular $\nabla^{a} r$ is outgoing everywhere thereon). Then, $P$ is empty, the maximum of $m$ on $\Sigma \cap B$ occurs on its boundary (which lies on $\mathcal{E}$ ) where it is no greater than $M_{\text {irr }}$. So, in this case, theorem 2 reproduces the bound given by part (b) of theorem 1. If, however, the spacetime contains no such Cauchy surface (see for example the black-hole spacetime given by Fig. (1), then $\max _{(\Sigma \cap B) \cup P}(m)$ can be larger than $\sup _{\mathcal{E}}\left(M_{\text {irr }}\right)$. As discussed in Sec. II, this larger bound is necessary. Furthermore, as $(\Sigma \cap B) \cup P$ is a subset of $\Sigma \backslash\langle\langle\mathcal{J}\rangle\rangle$, this quantity is constructed from the geometry on the portion of $\Sigma$ that is not in the asymptotic region. Lastly, we note that the rather complicated bound given by theorem 2 can always be replaced by the simpler but cruder bound $20 \sup _{\Sigma}(m)$ as $(\Sigma \cap B) \cup P$ is a subset of $\Sigma$ and $\sup _{\mathcal{E}}\left(M_{\mathrm{irr}}\right) \leq M_{\mathrm{ADM}} \leq \sup _{\Sigma}(m)$. (Note that this theorem suggests a strengthening of conjecture 2.)

The bound given by theorem 1 has the feature that it increases as matter flows through the event horizon into the black hole thereby making it larger. Therefore, it might seem that we could help an observer that has entered a black hole live longer by gathering up all the material available to us outside the black hole and throw it in after him. Does increasing the black-hole's mass in this way really help the observer? In Sec. \, we formulate and study a precise version of this problem. We find that while generally the lifetime of the observer can be increased, there are circumstances in which our effectiveness is limited and others in which there is nothing that can be done to help the unlucky traveler.

The remainder of this work is organized as follows. In Sec. II, we go through the steps leading to the formulation of conjectures 1 and 2. In Sec. III, we generalize the class of Cauchy surface topologies for which we believe conjecture 2 holds and prove a theorem which bounds the lengths of causal curves lying "deep within" a black hole in a spacetime possessing a maximal Cauchy surface. In Sec. IV, we provide the proofs of theorems 1 and 2. In Sec. V, we investigate the problem of whether we can increase the lifetime of an observer within a black hole. Lastly, in Sec. VI, we make a few final remarks and discuss the hopes for extending the results presented.

Our conventions are those of Ref. [4]. In particular, metrics are such that timelike vectors have negative norms and the Riemann and Ricci tensors are defined by $2 \nabla_{[a} \nabla_{b]} \omega_{c}=R_{a b c}{ }^{d} \omega_{d}$ and $R_{a b}=R_{a m b}{ }^{m}$, respectively. All metrics are taken to be $C^{2}$. For two sets $A$ and $B$, $A \backslash B$ denotes the elements in $A$ that are not in $B$ (i.e., the set difference), $A^{c}$ denotes the elements that are not in $A$ (i.e., its complement), and $\partial A$ denotes the boundary of $A$. Lastly, our units are such that $G=c=1$.

\section{FORMULATING AND TESTING CONJECTURES 1 AND 2}

An observer beginning a journey from the asymptotic region of a maximally extended Schwarzschild spacetime 
of positive mass $M$ (region I in Fig. 1) into the black hole (regions II and IV) must enter region II. As is well known, this journey within the black hole must end in a time no greater than $\pi M$ as the observer will be crushed as he approaches the singularity within. Does a similar result hold more generally? Consider the following conjecture.

Conjecture 3 (False). There is a constant $k$ such that the lifetime of any observer in a black hole of mass $M$ is no greater than $k M$.

We begin by testing this conjecture against the stationary (electrovacuum) black-hole spacetimes. For a KerrNewman black hole of mass $M$, angular momentum $M a$, and electric charge $e$, with $M^{2}>a^{2}+e^{2}>0$, we immediately realize that some care is needed in addressing this problem. Here, an observer can travel into the black hole and cross the Cauchy horizon associated with a Cauchy surface $\Sigma$ that extends to spacelike infinity at both ends. (See Fig. 28(i) of Ref. [3] or Fig. 12.4 of Ref. [4].) After crossing this horizon, he can travel to another asymptotically flat region and thereby live an indefinitely long time. However, that such a journey can be achieved is perhaps not surprising once we realize that the observer, having gone outside of the domain of dependence of $\Sigma$, is in a region that is unpredictable (from the initial data on $\Sigma$ ). It has been conjectured (the cosmic censorship conjecture [7, 10, 4] ) and investigations have indicated that such Cauchy horizons do not occur for "realistic" spacetimes [12,13. So, while a few spacetimes will have such horizons beyond which the journey of an observer could continue (such as Kerr-Newman with $M^{2}>a^{2}+e^{2}>0$ ) this will usually not be the case as the spacetime will become sufficiently singular that the journey will be inextendible. (Just how singular it will become has become a matter of debate [14,15].) It is because of such counterexamples that we have restricted ourselves to globally hyperbolic spacetimes in conjectures 1 and 2. Should either conjecture hold, we then have an upper bound on the time that an observer can remain within the domain of dependence of $\Sigma$ once inside the black hole. Whether the spacetime can be extended (so that the journey of the observer can continue) or otherwise (so that the journey is at an end) is then a matter for further investigation.

Restricting ourselves to the globally hyperbolic portion of the Kerr-Newman spacetimes (with Cauchy surfaces extending to spacelike infinity in both asymptotic regions) we find that conjecture 3 holds with $M$ being the irreducible mass associated with the event horizon (which is constant thereon) and $k \geq 2 \pi$. (Our reasons for identifying $M$ with $M_{\text {irr }}$ and not $M_{\mathrm{ADM}}$ or some other quantity will be given below.) Therefore, the KerrNewman spacetimes obey conjecture 1 provided $k \geq 2 \pi$. Furthermore, these spacetimes show that $2 \pi$ is the least value of $k$ for which the conjecture can hold as it can be shown that the ratio of the maximum lifetime of an observer within the portion of the black hole lying to the future of $\mathcal{J}^{-}$and $M_{\text {irr }}$, while always less than $2 \pi$, approaches $2 \pi$ in a sequence of Kerr-Newman black holes where $M^{2}-a^{2}-e^{2} \rightarrow 0$ (i.e., the ratio approaches $2 \pi$ in the limit that the black hole becomes extreme).

In conjecture 3 , no conditions are stated on the material content of the spacetime. Without such a condition we can easily counterexample the conjecture as follows. From an extended Schwarzschild spacetime of positive mass, construct a new spacetime by multiplying the Schwarzschild metric by a conformal factor that is unity outside the black-hole region (i.e. on regions I and III in Fig. 11). Then, as the two spacetimes share the same causal structure and agree outside the black-hole region of Schwarzschild, the share the same black-hole region. By choosing the conformal factor appropriately, we can quite easily ensure the existence of arbitrarily long timelike curves in the black-hole region (in particular, region II). We conjecture that spacetimes with "ordinary" matter do not exhibit this type of behavior. Just what restrictions on the matter content is appropriate is an issue for investigation, however, in conjectures 1 and 2 we have imposed the dominant-energy and non-negativepressures conditions [18]. As is demonstrated by theorems 1 and 2, together these conditions are sufficient for establishing a theorem in the spherically symmetric case. It is likely that these conditions can be weakened as the Kerr-Newman spacetimes satisfy the conclusions of conjecture 1 (with $k=2 \pi$ ) without satisfying the nonnegative-pressures condition (in the $e \neq 0$ case).

Further, in conjecture 3 there is no restriction on how the observer enters the black hole. This is important as the black-hole region associated with the asymptotic region I of Schwarzschild is all of regions II and IV in Fig. 1. In particular the other asymptotic end is inside the black hole (with our definition of black hole). So, clearly there are infinitely long lived observers therein. We need some type of condition to rule out this and similar counterexamples. Is it enough to restrict ourselves to observers that begin their journey from outside the black hole? No. Consider an observer that begins his journey in region III. While initially outside the black hole, he can go inside and reach the other asymptotic region and thereby live forever. However, an observer that begins in the asymptotic portion, region I, must enter region II upon entering the black hole wherein his lifetime must end in a finite time. So, one way to take care of this problem is to restrict ourselves to observers who enter the black hole from "sufficiently far away". For example, we can restrict ourselves to observers that begin their journey somewhere in the asymptotic region $\langle\langle\mathcal{J}\rangle\rangle$ (region I in Fig. 11) as we have done in conjecture 1. Or, somewhat more weakly, we can restrict ourselves to observers that lie to the future of $\mathcal{J}^{-}$. A second possibility is to restrict ourselves to spacetimes with $\mathbb{R}^{3}$ Cauchy surfaces as we have done in conjecture 2. This way, any possibility of escaping to another asymptotic region is eliminated $a b$ initio. (More general topologies are considered in Sec. III.)

Lastly, in conjecture 3, we have not specified what is meant by the mass $M$. Should we use the irreducible mass (as we have done in conjecture 1), the ADM mass 
of the spacetime, a quasilocal mass constructed on the event horizon of the black hole, a quantity constructed from the initial data (as we have done in conjecture 2), or something else? To investigate this issue, consider the black-hole spacetimes associated with the gravitational collapse of uniform density "dust ball" such as that depicted in Fig. 3. (These are the spacetimes considered by Oppenheimer and Snyder in their pioneering work on gravitational collapse 333.) These dust-ball spacetimes are easily constructed by "gluing" a portion of a dustfilled Robertson-Walker spacetime to Schwarzschild [34] and we refer the reader to Appendix B for a brief review of their construction and the establishment of notation for the following.

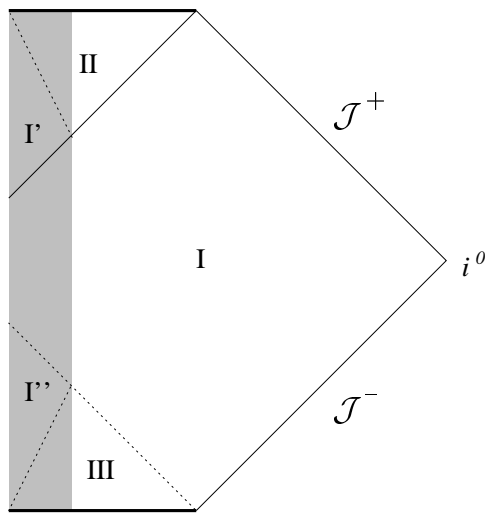

FIG. 3. A dust-ball spacetime constructed from a $k_{\text {RW }}=+1$ Robertson-Walker spacetime with $0<\chi_{0}<\pi / 3$. The black-hole region here is the union of regions $\mathrm{I}^{\prime}$ and II.

Note that for these spacetimes, the mass function $m$ coincides with the ADM mass in the vacuum region and since all of the matter eventually goes into the black hole, $M_{\text {irr }}$ and $m$ both have $M_{\mathrm{ADM}}$ as future asymptotic limits on the event horizon. So, while these spacetimes cannot distinguish which of these three quantities should be used in conjecture 3, they can test whether one (and hence all) will fail to work. To see why all three are inappropriate for conjecture 2, fix a dust-ball spacetime constructed from a $k_{\mathrm{RW}}=+1$ Robertson-Walker spacetime (so the dust ball is gravitational bound) that is described by the

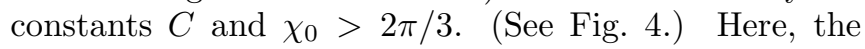
black hole is the union of regions $\mathrm{I}^{\prime \prime \prime}, \mathrm{II}, \mathrm{II}^{\prime}, \mathrm{III}^{\prime}$, and IV.

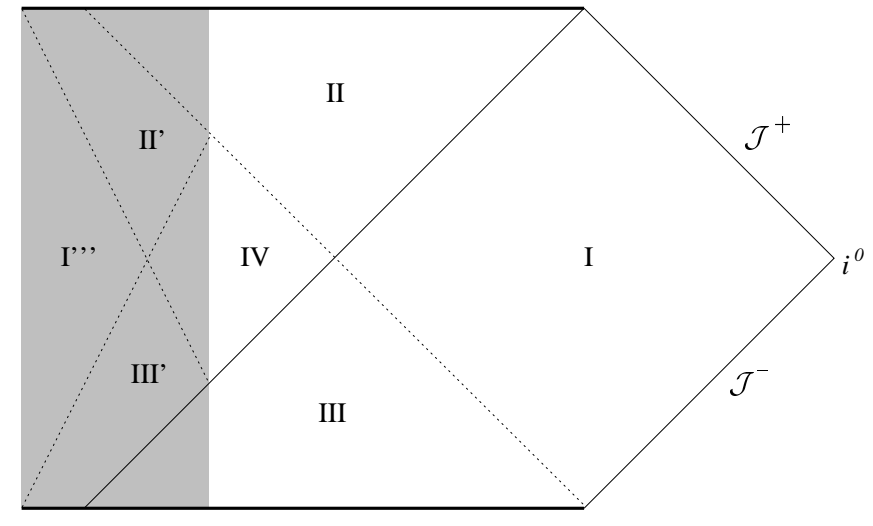

FIG. 4. A dust-ball spacetime constructed from a $k_{\mathrm{RW}}=+1$ Robertson-Walker spacetime with $2 \pi / 3<\chi_{0}<\pi$. The black hole here is the union of regions $\mathrm{I}^{\prime \prime \prime}, \mathrm{II}, \mathrm{II}^{\prime}, \mathrm{III}^{\prime}$, and IV. An observer that begins his journey in region I and enters the black hole, must enter region II. On the other hand, an observer beginning his journey from region III has the freedom to visit all of the black-hole regions. Notice that in this case every Cauchy surface must intersect the black hole and that no spherically symmetric Cauchy surface exists on which $\nabla^{a} r$ is outgoing everywhere thereon.

For observers within the black hole, not necessarily entering from region I, it can be argued that the maximum lifetime that can be spent within the black hole is attained by the curve corresponding to the center of the dust ball. Therefore,

$$
\begin{aligned}
\text { (maximum lifetime) } & =\int_{0}^{2 \pi} a(\eta) d \eta \\
& =\pi C \\
& =\left(\frac{2 \pi}{\sin ^{3} \chi_{0}}\right) M_{\mathrm{ADM}} .
\end{aligned}
$$

Now consider a sequence of such spacetimes in which $\chi_{0} \rightarrow \pi$. In this limit, the coefficient of $M_{\mathrm{ADM}}$ in Eq. (2.1d) diverges. Therefore, this sequence of spacetimes shows that, provided that we always identify $M$ as the ADM mass of the spacetime (or the future asymptotic values of $M_{\text {irr }}$ or $m$ on the event horizon), there is no constant $k$ for which the above conjecture will hold. This is not surprising once we realize that in such a sequence (with fixed $C$ ) the maximum lifetime of observers inside the black hole, given by Eq. (2.1b), is fixed, while the mass of the black hole, as measured using the ADM mass given by Eq. (B6) is becoming arbitrarily small in this limit. This shows, in particular, that an upper bound on the lifetime of observers inside of a black hole cannot, in general, be determined by examining the spacetime outside of the black hole. With $\chi_{0}$ close to $\pi$, the size of the spheres of symmetry inside the black hole and the amount of matter therein can be much greater than would be guessed by examining the mass of the black hole from the outside. Indeed, the spacetime within the black hole acts very much like a closed universe (in particular like a $k_{\mathrm{RW}}=+1$ Robertson-Walker spacetime) except 
that it is connected to an asymptotically flat spacetime by a small "throat".

So, conjecture 2 would fail if we identified $M_{\Sigma}$ with $M_{\mathrm{ADM}}$ or the future asymptotic values of $M_{\text {irr }}$ or $m$ on the event horizon. Is there some other quantity that we can construct that is sensitive to the geometry inside of the black hole? Note that for these spacetimes with $k_{\mathrm{RW}}=+1$ and $\chi_{0}>\pi / 2$, the mass $m$ is not maximal on the outer boundary of the dust ball. It is maximal on the surface $\chi=\pi / 2$. Fixing a spherically symmetric Cauchy surface $\Sigma$ in our spacetime and using Eq. (B5), we see that the supremum of $m$ over $\Sigma$ is simply $C / 2$. Therefore, by Eq. (2.1b), for these spacetimes we have

$$
(\text { maximum lifetime })=2 \pi \sup _{\Sigma}(m) .
$$

So, conjecture 2 does hold for these spacetimes provided that we take $M_{\Sigma}$ to be the supremum of $m$ over a Cauchy surface and $k \geq 2 \pi$. Analyzing the remaining dust-ball spacetimes, we again find that conjecture 2 holds provided that $k \geq 4+\pi$ and $M_{\Sigma}=\sup _{\Sigma}(m)$. (Note that this lower bound on the allowed values of $k$ is not least. Finding the least upper bound to the lengths of timelike curves in the black holes of the dust-ball spacetimes with $k_{\mathrm{RW}}=-1$ or $k_{\mathrm{RW}}=+1$ and $\chi_{0}<\pi / 2$ does not appear to be an easy task. For instance, is the geodesic at the center of the dust ball longest? With some work it can probably be shown that the conjecture holds for all of these spacetimes with $k \geq 2 \pi$.)

From these observations and in view of theorem 2, it would seem that the proper identification for $M_{\Sigma}$ conjecture 2 would be something that generalizes $\sup _{\Sigma}(m)$. The most obvious candidate is to generalize $m$ by some quasilocal mass function and then find its supremum over the set of all two-spheres on a Cauchy surface $\Sigma$. However, whether this is truly appropriate we shall leave as a matter for future investigation (i.e., finding such a quantity for conjecture 2 and proving it, or somehow show that there is no such notion for which conjecture 2 will hold).

Analyzing the dust-ball spacetimes $\left(k_{\mathrm{RW}}=+1, k_{\mathrm{RW}}=\right.$ 0 , and $k_{\mathrm{RW}}=-1$ ) we find that conjecture 1 always holds with $k \geq 4+\pi$. (Again, this lower bound on $k$ is not least and it is likely that the conjecture holds for all $k \geq 2 \pi$.) So, from these observations, it would seem that $M_{\mathrm{ADM}}$ or the future asymptotic values of $M_{\text {irr }}$ or $m$ could be appropriate for the mass in conjecture 1. It is in the proof of conjecture 1 in the spherically symmetric case (i.e., theorem 1) that we discover that $M_{\text {irr }}$ is the most natural candidate, which is why this is the quantity chosen for conjecture 1 .

\section{GENERALIZING THE TOPOLOGY IN CONJECTURE 2}

In this section we investigate the problem of relaxing the restriction to $\mathbb{R}^{3}$ Cauchy surfaces in Conjecture 2 .
As we are interested in asymptotically flat spacetimes, we begin by restricting ourselves to manifolds where all of the "interesting topology" is bounded away from infinity. We make this idea precise by demanding that the Cauchy surfaces have the topology of a connected, orientable, three-manifold without boundary $C$ less a point which we label $i^{0}$. The idea here is that deleted point $i^{0}$ "represents" spatial infinity. So, outside of a sufficiently large sphere (i.e., inside of a two-sphere sufficiently near $i^{0}$ ) the space has the topology $\mathbb{R} \times S^{2}$. Further, to exclude the possibility of escaping to other asymptotic regions, as in the maximally extended Schwarzschild spacetime, we demand that $C$ be compact. This way all of the non-compactness of a Cauchy surface $\Sigma$ is due to the single asymptotic region. We summarize these requirements by demanding that $\Sigma \approx C \backslash i^{0}$, where $C$ is a connected, orientable, closed (compact without boundary) three-manifold. As a simple example, $\Sigma \approx \mathbb{R}^{3}$ is of this form with $C \approx S^{3}$.

Restricting ourselves to spacetimes with Cauchy surfaces diffeomorphic to $C \backslash i^{0}$, does conjecture 2 hold for all closed three-manifolds $C$ ? Remarkably, the answer is no. We can construct a spacetime meeting the conditions of Conjecture 2 with $C=T^{3}=S^{1} \times S^{1} \times S^{1}$ (the three-torus) and yet possessing infinitely long timelike curves within the black hole as follows 35. In constructing the $k=0$ dust-ball spacetimes, we took the region lying inside of a spherically symmetric timelike three-surface in a $k=0$ Robertson-Walker spacetime and attached it to Schwarzschild as we wanted the model to have $\mathbb{R}^{3}$ Cauchy surfaces. Taking the region lying outside of this timelike three-surface and attaching it to Schwarzschild, we obtain a globally hyperbolic asymptotically flat spherically symmetric spacetime with $\mathbb{R} \times S^{2}$ Cauchy surfaces and infinitely long lived observers within the black hole-just like in Schwarzschild. The spatial topology still has the form $C \backslash i^{0}$, although $C$ here being $\mathbb{R}^{3}$ is not compact. However, the spatial homogeneity of the Robertson-Walker spacetimes allows an alternative construction that gives us black-hole spacetimes with $C$ closed. From a $k=0$ Robertson-Walker spacetime we construct a new spacetime by simply identifying the the spacetime under a discrete group of isometries so that the spatial topology is now $T^{3}$. Now choosing a (locally spherically symmetric) two-sphere in this spacetime and generating a timelike three-surface by carrying the sphere along the flow of the dust and then attaching the exterior of this three-surface appropriately to a portion of maximally extended Schwarzschild, we again have a globally hyperbolic asymptotically flat spacetime, but now the Cauchy surfaces are diffeomorphic to $T^{3}$ minus a point. This spacetime admits infinitely long timelike curves in the black-hole region and therefore, independent of what is chosen for $M_{\Sigma}$ (as long as it finite) there is no finite $k$ for which conjecture 2 would hold for Cauchy surfaces diffeomorphic to $T^{3} \backslash i^{0}$. (Note that we can perform a similar construction on the $k=-1$ Robertson-Walker spacetimes.) 
So, as conjecture 2 does not hold for all Cauchy surfaces of the form $C \backslash i^{0}$ with $C$ closed, are there any restrictions on the allowed topologies of $C$ that will give a generalized Conjecture 2 a chance to hold? A hint is provided by the realization that in the study of the closed-universe recollapse conjecture, a restriction on the Cauchy surface topology is necessary as otherwise there may be a topological obstruction to the existence of a maximal Cauchy surface. Furthermore, in the case where a maximal Cauchy surface exists, there is a finite upper bound to the lengths of all causal curves in the spacetime (provided certain energy and genericity conditions are met) [32]. Does a similar result hold for the asymptotically flat spacetimes? That is, if the spacetime does admit a maximal Cauchy surface, then must there be an upper bound to the lengths of timelike curves within the black-hole region? Whether this is the case is unknown, however, adapting the ideas of Bartnik [36, 37], we can bound the lengths of timelike curves in the portion of the black hole that is not reachable from the asymptotic region.

Consider the portion of the black hole that is so deep within that it is "hidden" in the sense that it cannot be reached from $\langle\langle\mathcal{J}\rangle\rangle$, i.e.,

$$
M \backslash\left(J^{-}\left(\mathcal{J}^{+}\right) \cup J^{+}\left(\mathcal{J}^{-}\right)\right) .
$$

This region is globally hyperbolic (provided the entire spacetime is globally hyperbolic) and is simply the intersection of the black-hole and white-hole regions associated with an asymptotic end. In Schwarzschild, this is all of region IV in Fig. 1. In the dust-ball spacetime of Fig. 3, this set is empty. In the dust-ball spacetime of Fig. 1, this is the union of regions $\mathrm{I}^{\prime \prime \prime}, \mathrm{II}^{\prime}, \mathrm{III}^{\prime}$, and IV. Note that this is precisely the region of the black hole that is not covered by conjecture 1. Furthermore, for the spacetimes with Cauchy surfaces diffeomorphic to a closed threemanifold minus a point, these regions are very much like closed universes in that for any Cauchy surface $\Sigma$, the domain of dependence of the compact set $\Sigma \backslash\langle\langle\mathcal{J}\rangle\rangle$ contains the hidden black-hole region. Therefore, the evolution of these regions is determined by data on a compact set. This property provides another connection between the conjectures that observers in closed-universes and blackhole regions must have finite lifetimes and using it we now show that if the spacetime admits a maximal Cauchy surface, then the lengths of all causal curves in this hidden portion of the black hole are bounded above.

Theorem 3. Fix an asymptotically flat spacetime satisfying the timelike-convergence condition [18] and possessing a maximal Cauchy surface $\Sigma_{0}$ diffeomorphic to $C \backslash i^{0}$ where $C$ is a connected, orientable, closed three-manifold. Then, there exists an upper bound on the lengths of all causal curves in the hidden portion of the black hole (i.e., the region given by Eq. (3.1)).

Proof. Denoting the hidden portion of the black hole by $H$, our task is to show that $d(H, H)$ is finite. Since $d(H, H) \leq d\left(H, \Sigma_{0}\right)+d\left(\Sigma_{0}, H\right)$, we need only show that
$d\left(H, \Sigma_{0}\right)$ and $d\left(\Sigma_{0}, H\right)$ are finite. [Recall that $d(p, q)$ is the distance function defined as the least upper bound to the length of all continuous causal curves connecting $p$ to $q$ if $q \in J^{+}(p)$, and zero otherwise, and $d(\mathcal{P}, \mathcal{Q})$ is defined for subsets $\mathcal{P}$ and $\mathcal{Q}$ of $M$ as the least upper bound of $d(p, q)$ over all $p \in \mathcal{P}$ and $q \in \mathcal{Q}[3]$.]

We bound $d\left(\Sigma_{0}, H\right)$ as follows. Fix any point $p \in$ $D^{+}\left(\Sigma_{0}\right) \cap H$ and let $\gamma$ be a longest timelike curve from $\Sigma_{0}$ to $p$. As is well known, $\gamma$ intersects $\Sigma_{0}$ orthogonally, is geodetic, and has no points between $\Sigma_{0}$ and $p$ conjugate to $\Sigma_{0}$. (See, for example, Sec. 9.3 of Ref. [1].) However, this last requirement is difficult to satisfy for long curves as a congruence of timelike geodesics meeting $\Sigma_{0}$ orthogonally has vanishing expansion on $\Sigma_{0}$ since $\Sigma_{0}$ has zero trace extrinsic curvature $K$ by virtue of its being a maximal hypersurface. Were $K$ everywhere bounded away from zero by a negative constant $-\kappa$ on $\Sigma_{0}$, or merely on $J^{-}(H) \cap \Sigma_{0}$, then the length of $\gamma$ (which equals $d\left(\Sigma_{0}, p\right)$ ) could be no larger than $3 / \kappa$. (See theorem 9.5.1 of Ref. [. 1 .) However, $K$ is merely zero on $\Sigma_{0}$. To take care of this problem, we construct a Cauchy surface $\Sigma_{1}$ to the future of $\Sigma_{0}$ having negative trace extrinsic curvature bounded away from zero on $J^{-}(H) \cap \Sigma_{1}$ by "pushing" or "evolving" $\Sigma_{0}$ to the future.

We construct $\Sigma_{1}$ by first constructing a family of Cauchy surfaces $\Sigma_{t}$ by evolving $\Sigma_{0}$ with zero shift (so the displacement is purely normal) and non-constant lapse $N$. The trace of the extrinsic curvature of the surface $\Sigma_{t}$ then evolves according to

$$
\frac{\partial K}{\partial t}=-N\left(K_{a b} K^{a b}+R_{a b} n^{a} n^{b}\right)+D_{a} D^{a} N,
$$

where $n_{a}=-N(d t)_{a}$ is the future-directed unit normal to the surfaces, $K_{a b}$ is the extrinsic curvature of the surfaces, and $D_{a}$ is the derivative operator associated with the induced metric on each surface. Note that with $N=1$, the right-hand side of Eq. (3.2) is always non-negative as $K_{a b} K^{a b}$ is manifestly non-negative and $R_{a b} n^{a} n^{b}$ is non-negative by the timelike-convergence condition. Were the sum of these quantities positive everywhere on $\Sigma_{0}$, then by simply evolving $\Sigma_{0}$ with $N=1$, we would have a Cauchy surface with $K<0$ for any $t>0$. However, this need not be the case, so we need to be a bit more subtle in our choice of $N$.

Let $Y_{0}$ be any compact subset of $\Sigma_{0}$ (with smooth boundary) whose interior contains the compact set $X_{0}=$ $\Sigma_{0} \backslash J^{+}\left(\mathcal{J}^{-}\right)$. Then, $\left(J^{-}(p) \cap \Sigma_{0}\right) \subset X_{0} \subset Y_{0}$. There is a great deal of freedom in our construction of $\Sigma_{t}$ and hence $\Sigma_{1}$, but for definiteness, we shall consider any family of hypersurfaces $\Sigma_{t}$ that arises from $\Sigma_{0}$ and a lapse that obeys the property that, on $\Sigma_{0}, N$ is the unique solution of the elliptic partial differential equation $D_{a} D^{a} N=-f$ for some scalar field $f$ that is positive in the interior of $Y_{0}$ and zero on $\Sigma_{0} \backslash Y_{0}$ and that satisfies the boundary condition that $N$ be zero on $\Sigma_{0} \backslash Y_{0}$. By Hopf's maximum principle [38], $N$ is positive everywhere in the interior of $Y_{0}$, so by Eq. (3.2), $\partial K / \partial t \leq-f$ on $Y_{0}$ and therefore $\partial K / \partial t \leq-c$ on $X_{0}$ for some positive constant $c$. 
So, for sufficiently small $t$, on $\Sigma_{t} \backslash J^{+}\left(\mathcal{J}^{-}\right)$the trace of the extrinsic curvature $K$ is bounded away from zero by the negative constant $-\kappa=-c t$. We shall take $\Sigma_{1}$ to be any such surface. So, as $J^{-}(p) \cap \Sigma_{1}$ is a subset of of $\Sigma_{1} \backslash J^{+}\left(\mathcal{J}^{-}\right)$and $K \leq-\kappa$ thereon, by the argument above, $d\left(\Sigma_{1}, p\right) \leq 3 / \kappa$ and therefore $d\left(\Sigma_{1}, H\right) \leq 3 / \kappa$. Noting that $d\left(\Sigma_{0}, H\right) \leq d\left(\Sigma_{0}, \Sigma_{1}\right)+d\left(\Sigma_{1}, H\right)$ and the fact that $d\left(\Sigma_{0}, \Sigma_{1}\right)$ is finite (as $\Sigma_{0}$ and $\Sigma_{1}$ coincide outside of compact sets) we have established the existence of an upper bound on $d\left(\Sigma_{0}, H\right)$.

By a time-reversed argument, a similar bound can be established on $d\left(H, \Sigma_{0}\right)$. Therefore, we have established the existence of a finite upper bound on $d(H, H)$ as was to be shown.

So, should the spacetime admit a maximal Cauchy surface, then there will not be arbitrarily long timelike curves contained within the hidden portion of the black hole. While sufficient conditions have been given for the existence of such surfaces 39.40], not all asymptotically flat spacetimes admit maximal Cauchy surfaces. Indeed, the Cauchy surface topology may provide an obstruction to the existence of such a surface. This follows from the simple fact that scalar constraint equation of general relativity demands that the Ricci scalar curvature associated with the induced metric on such a surface must be non-negative provided the spacetime satisfies the non-negative-energy condition [18] while most threemanifolds of the form $C \backslash i^{0}$ do not admit Riemannian metrics with non-negative scalar curvature [41]. For instance, our $T^{3} \backslash i^{0}$ black-hole spacetime above does not admit a maximal Cauchy surface because of this obstruction.

What three-manifolds $C \backslash i^{0}$ (with $C$ as before) admit asymptotically flat metrics of non-negative scalar curvature, i.e., those that do not provide a topological obstruct the existence of a maximal Cauchy surface? Witt has shown that any such manifold $C$ also admits a Riemannian metric with positive scalar curvature 442]. It then follows from the work of Gromov and Lawson that $C$ must be $S^{3}, S^{1} \times S^{2}$, or a manifold that can be constructed from one of these by making certain identifications and connected summations [43]. Therefore, we generalize the Cauchy surface topology in conjecture 2 to include each of these manifolds less a point.

While theorem 3 does support the plausibility of conjecture 2 (in that some bound should exist), it would be nice to strengthen its results beyond the hidden portion of the black hole. However, with the current method of proof, it does not appear that such a generalization is possible. The problem is that $I^{-}(B) \cap \Sigma_{0}$ need not be contained in a compact subset of $\Sigma_{0}$ and therefore the construction of a Cauchy surface $\Sigma_{1}$ that is contracting at a rate bounded away from zero on subset that acts as a Cauchy surface for the region of the black hole lying to the future of $\Sigma_{1}$ will fail. The dust-ball spacetimes in Figs. 3 and 1 provides simple examples of this behavior. However, in these spacetimes, for a timelike curve in the black-hole region, the closure of $I^{-}(\gamma) \cap \Sigma_{0}$ is a compact subset of $\Sigma_{0}$. So, here we could show that there are no infinite length causal curve (although we would not have an upper bound independent of the curve). Can this weaker result be shown to hold? No. There is no (known) reason why $I^{-}(\gamma)$ must meet a Cauchy surface within a compact subset thereof as happens when $\gamma$ "runs into" a Cauchy horizon (as seen in Kerr-Newman). Lastly, we note that the lengths of causal curves in the region of the black hole $B$ lying to the past of $\Sigma_{0}$ are bounded as $J^{+}(B) \cap \Sigma_{0}$ is the compact set $B \cap \Sigma_{0}$. So, a timelike curve in a black-hole region of a spacetime satisfying the timelike-convergence condition and admitting a maximal Cauchy surface cannot have infinite length to the past.

\section{THE SPHERICALLY SYMMETRIC CASE}

Our strategy in proving theorems 1 and 2 is quite simple. The idea is to first establish a number of properties of $r$ and $m$ on the event horizon. Next, these properties are used to establish an upper bound for $r$ in the blackhole region (or just in the part lying to the future of $\mathcal{J}^{-}$). Once this is done, the theorem follows quite easily with the aid of the following lemma established earlier in the analysis of the closed-universe recollapse conjecture (and whose proof is essentially the subject of Sec. III of Ref. [28]).

Lemma 1. In a globally hyperbolic spherically symmetric spacetime satisfying the non-negative-pressures condition [18] and on which $m$ is everywhere non-negative and $r$ is everywhere bounded above by some constant $r_{U}$, the lengths of all timelike curves are bounded above by $10 r_{U}$.

In Sec. IV A, the elementary features of the spherically symmetric spacetime are reviewed. In Sec. IVB, some properties of $r$ and $m$ associated with asymptotic flatness are established. In Sec. IV hole region are established in a variety of circumstances. Lastly, in Sec. IVD, the proofs of theorem 1 and 2 are completed.

\section{A. Elementaries}

In this section, the elementary features of the spherically symmetric spacetimes needed here are briefly reviewed. For a more complete presentation, see Ref. [26].

A spacetime $\left(M, g_{a b}\right)$ is said to be spherically symmetric if it admits a group $G \approx \mathrm{SO}(3)$ of isometries, acting effectively on $M$, each of whose orbits is either a twosphere or a point. Denote the orbit of a point $p$ by $\mathcal{S}_{p}$. The value of the non-negative scalar field $r$ at each $p \in M$ is defined so that $4 \pi r^{2}$ is the area of $\mathcal{S}_{p}$. So, in particular, $r(p)=0$ if $\mathcal{S}_{p}=p$, while $r(p)>0$ if $\mathcal{S}_{p}$ is a two-sphere. Furthermore, we shall say that $\mathcal{S}$ is a sphere of symmetry if $\mathcal{S}=\mathcal{S}_{p}$ for some $p \in M$ and $\mathcal{S}$ is a two-sphere. 
Where $r>0$, we decompose the metric $g_{a b}$ into the sum $g_{a b}=h_{a b}+q_{a b}$, where $q_{b}^{a}$ is the projection operator onto the tangent space of each sphere of symmetry and $h^{a}{ }_{b}$ is the projection operator onto the tangent space of each two-surface perpendicular to the spheres of symmetry. Using the fact that there exists a preferred "unitmetric" $\Omega^{a b}$ on each sphere of symmetry, we have $q_{a b}=$ $r^{2} \Omega_{a b}$ (where $\Omega^{a m} \Omega_{m b}=q_{b}^{a}$ and $\Omega_{a b}=q^{m}{ }_{a} q_{b}^{n} \Omega_{m n}$ ). This gives us the final decomposition of $g_{a b}$ as

$$
g_{a b}=h_{a b}+r^{2} \Omega_{a b} .
$$

For the spherically symmetric spacetimes, the mass $m$ is defined by

$$
2 m=r\left(1-\nabla_{m} r \nabla^{m} r\right) .
$$

Defining $\epsilon^{a b}$ to be either of the two antisymmetric tensor fields such that $\epsilon^{a b} \epsilon^{c d}=-2 h^{a[c} h^{d] b}$, and denoting the "radial part" of the Einstein tensor $G^{a b}$ by $\tau^{a b}$ (i.e., $\tau^{a b}=$ $h^{a}{ }_{m} h_{n}^{b} G^{m n}$ ) we have

$$
h_{a}{ }^{m} h_{b}{ }^{n} \nabla_{m} \nabla_{n} r=\frac{m}{r^{2}} h_{a b}-\frac{r}{2} \tau^{m n} \epsilon_{m a} \epsilon_{n b},
$$

from which it follows that

$$
\nabla_{a}(2 m)=r^{2} \tau^{m n} \epsilon_{m a} \epsilon_{n b} \nabla^{b} r
$$

As the space of vectors perpendicular to the spheres of symmetry is two-dimensional and the metric thereon is Lorentzian, we shall label a radial vector as being either outgoing, ingoing, future-directed, or past-directed according to which quadrant the vector lies with the convention that $\nabla^{a} r$ is outgoing near $\mathcal{J}$. Radial null vectors will have two such labels (e.g., outgoing and futuredirected) while the zero vector will have all four. (In other words, the set of vectors under a particular label is closed.) Note that these designations are well defined globally for any spherically symmetric spacetime that is simply connected as are the spherically symmetric spacetimes with $\mathbb{R}^{3}$ or $\mathbb{R} \times S^{2}$ Cauchy surfaces.

\section{B. Properties of $r$ and $m$ associated with asymptotic flatness}

Our first lemma establishes a few basic properties of $r$.

Lemma 2. Fix a globally hyperbolic asymptotically flat spherically symmetric spacetime satisfying the nullconvergence condition [18]. Then: (a) on $J^{-}\left(\mathcal{J}^{+}\right), \nabla^{a} r$ cannot be future-directed or ingoing; (b) on $J^{+}\left(\mathcal{J}^{-}\right)$, $\nabla^{a} r$ cannot be past-directed or ingoing; (c) on $\langle\langle\mathcal{J}\rangle\rangle$, $\nabla^{a} r$ must be outgoing spacelike; (d) on $\partial J^{-}\left(\mathcal{J}^{+}\right), \nabla^{a} r$ must be either past-directed or outgoing and $r$ is nondecreasing to the future.

Proof. To prove (a), fix any sphere of symmetry $\mathcal{S} \subset J^{-}\left(\mathcal{J}^{+}\right)$. If $\nabla^{a} r$ where future-directed or ingoing on $\mathcal{S}$, then $\mathcal{S}$ would be a future outer trapped surface in $J^{-}\left(\mathcal{J}^{+}\right)$which is impossible by Proposition 9.2.8 of Ref. [3]. Part (b) follows from (a) by simply reversing the time-orientation of the spacetime. Part (c) follows from (a) and (b) and noting that outgoing spacelike is the only possibility left. That $\nabla^{a} r$ must be either pastdirected or outgoing in (d) follows from part (a) and continuity from which it follows that $r$ is non-decreasing to the future. (Alternatively, part (d) follows from Hawking's black-hole area theorem.) $\square$

Our next lemma establishes properties of $m$ in the asymptotic region and on the event horizon.

Lemma 3. Fix a globally hyperbolic asymptotically flat spherically symmetric spacetime satisfying the dominantenergy condition [18]. Then, on $\langle\langle\mathcal{J}\rangle\rangle, m \leq M_{\mathrm{ADM}}$. Further, on the portion of the event horizon lying the future of $\mathcal{J}^{-}$[i.e., $\left.\left(\partial J^{-}\left(\mathcal{J}^{+}\right)\right) \cap J^{+}\left(\mathcal{J}^{-}\right)\right], m$ is non-decreasing to the future and has a future limit which is no greater than $M_{\mathrm{ADM}}$.

Proof. As $\nabla^{a} r$ is outgoing spacelike on $\langle\langle\mathcal{J}\rangle\rangle$ by lemma $2, \nabla^{a} m$ is outgoing thereon by Eq. (4.4). Fix any point $p \in\langle\langle\mathcal{J}\rangle\rangle$, and a spherically symmetric Cauchy surface $\Sigma$ containing $p$. As $m$ is non-decreasing outwards on $\Sigma \cap\langle\langle\mathcal{J}\rangle\rangle$ and since $m \rightarrow M_{\mathrm{ADM}}$ as we approach $i^{0}$ in $\Sigma$, $m \leq M_{\mathrm{ADM}}$ at $p$ and hence on all of $\langle\langle\mathcal{J}\rangle\rangle$. By lemma 2, $\nabla^{a} r$ must be outgoing (and yet not past-directed) on $\left(\partial J^{-}\left(\mathcal{J}^{+}\right)\right) \cap J^{+}\left(\mathcal{J}^{-}\right)$, and hence $\nabla^{a} m$ must be outgoing thereon. Therefore, $m$ must be non-decreasing to the future thereon. By continuity, $m$ is bounded by $M_{\mathrm{ADM}}$ on $\left(\partial J^{-}\left(\mathcal{J}^{+}\right)\right) \cap J^{+}\left(\mathcal{J}^{-}\right)$and so $m$ must have a limit no greater than $M_{\mathrm{ADM}}$ to the future.

It is worth noting that the upper bound of $M_{\mathrm{ADM}}$ on $m$ on the event horizon need not hold everywhere on the event horizon. A simple example is provided by the dustball spacetime in Fig. A. However, on the portion of the event horizon lying to the future of some Cauchy surface, $m$ is bounded as follows.

Lemma 4. Fix a globally hyperbolic asymptotically flat spherically symmetric spacetime satisfying the nonnegative-pressures condition [18]. Fix a Cauchy surface $\Sigma$ therein and denote by $P$ the subset of $\Sigma$ on which $\nabla^{a} r$ is past-directed timelike, past-directed null, or zero. On the portion of the black-hole's event horizon $\mathcal{E}$ in the future domain of dependence of $\Sigma$, i.e., $D^{+}(\Sigma) \cap \mathcal{E}, m$ is bounded above by the expression

$$
2 m \leq \max \left(\sup _{\mathcal{E}}(r), \sup _{P}(2 m)\right) .
$$

Proof. By lemma $2, \nabla^{a} r$ is either outgoing or pastdirected on $\mathcal{E}$. Where $\nabla^{a} r$ is outgoing, then $2 m \leq r \leq$ $\sup _{\mathcal{E}}(r)$, where the first inequality follows directly from the definition of $m$. Where $\nabla^{a} r$ is past-directed, by lemma $\mathrm{C} 1$ of Appendix C, $2 m \leq \sup _{P}(2 m)$. Combining these results, Eq. (4.5) follows.

Lastly, that $m$ is everywhere non-negative for the spacetimes under consideration is given by the following lemma.

Lemma 5. On a spherically symmetric spacetime satisfying the dominant-energy condition [18] and admitting 
a complete spherically symmetric Cauchy surface $\Sigma, m$ is everywhere non-negative.

Proof. We first show that $m$ is non-negative on $\Sigma$ and then show that the non-negativity of $m$ everywhere else follows. Suppose that at some point $p \in \Sigma$ that $m(p)<0$. Then, by Eq. (4.2), $\nabla^{a} r$ is necessarily spacelike at $p$. Denote the subset of $\Sigma$ where $r>0$ by $\tilde{\Sigma}$ (which is just $\Sigma$ less one, two, or possibly no points) and let $s^{a}$ the spherically symmetric unit-vector field on $\tilde{\Sigma}$ such that $s^{a} \nabla_{a} r<0$ at $p$. Consider the maximal integral curve $\sigma$ of $s^{a}$ emanating from $p$. Then, as $s^{a} \nabla_{a}(2 m) \leq 0$ where $\nabla^{a} r$ is spacelike with $s^{a} \nabla_{a} r<0, m$ is negative and $\nabla^{a} r$ is spacelike all along $\sigma$. Therefore, along $\sigma$, the change in $r$ with respect to the distance $s$ measured along $\sigma$ from $p$ is bounded by $d r / d s=s^{a} \nabla_{a} r \leq-\sqrt{\nabla^{a} r \nabla_{a} r}=$ $-\sqrt{1-2 m / r}<-1$. Integrating this and using the fact that $r>0$ on $\tilde{\Sigma}$, the length of $\sigma$ is bounded above by $r(p)$. The curve $\sigma$ is geodetic (in $\Sigma$ ) and, furthermore, it cannot be extended to a greater length in $\Sigma$ as $m$ is zero where $r$ is zero. Therefore, with $\Sigma$ complete, $m$ must be non-negative everywhere on $\Sigma$.

Having established that $m$ is non-negative on $\Sigma$, we now show that $m$ is non-negative everywhere as follows. Suppose that $m<0$ at some point $p \in D^{+}(\Sigma)$. Then, $\nabla^{a} r$ is necessarily spacelike at $p$. So, consider the pastdirected radial null geodesic $\nu$ with future endpoint $p$ such that $k^{a} \nabla_{a} r<0$ at $p$, where $k^{a}$ is a past-directed tangent vector to $\nu$. Then, either: (i) $\nabla^{a} r$ remains spacelike with $r>0$ on $\nu$ in which case it must intersect $\Sigma$ at some point $q$. So, as $k^{a} \nabla_{a}(2 m) \leq 0$, we have $m(p) \geq m(q) \geq 0$; or (ii) $\nabla^{a} r$ remains spacelike on $\nu$ but it intersects the "center of symmetry" where $r=0$ at some point $q$, so that $m(p) \geq m(q)=0$; or (iii) at some point $q$ on $\nu$, $\nabla^{a} r$ fails to remain spacelike in which case it must be null or zero so that $m(p) \geq m(q)=r(q) / 2>0$. In all three cases, $m(p)$ is non-negative, so $m$ is non-negative on $D^{+}(\Sigma)$. That $m$ is non-negative on $D^{-}(\Sigma)$ follows by a similar argument.

\section{Bounding $r$ inside the black hole}

Here we establish bounds on $r$ in the black-hole region under a variety of circumstances.

Lemma 6. Fix a globally hyperbolic asymptotically flat spherically symmetric spacetime satisfying the nullconvergence condition [18] and possessing a black hole $B$ with event horizon $\mathcal{E}=\partial B$. Fix a spherically symmetric Cauchy surface $\Sigma$ for this spacetime and denote by $\Sigma^{\prime}$ the component of $\Sigma$ connected to $i^{0}$ on which $\nabla^{a} r$ is outgoing. Then,

$$
r \leq \sup _{\mathcal{E}}(r)
$$

on: (a) that portion of the black hole lying in the future of the past null-infinity [i.e., on $B \cap J^{+}\left(\mathcal{J}^{-}\right)$]; (b) on the portion of the black hole lying in the future of $\Sigma^{\prime}$ [i.e., on
$\left.B \cap J^{+}\left(\Sigma^{\prime}\right)\right]$; (c) on all of the black-hole region provided that $\nabla^{a} r$ is outgoing on all of $\Sigma$.

Proof. To prove (a), fix any point $p \in B \cap J^{+}\left(\mathcal{J}^{-}\right)$. Let $\nu$ be an outgoing null geodesic generator of $\partial J^{-}\left(\mathcal{S}_{p}\right)$. Then $\nu$ has a future endpoint on $\mathcal{S}_{p}$ and no past endpoint. Further, it can be shown that $\nu$ is contained in $J^{+}\left(\mathcal{J}^{-}\right)$ and enters $\langle\langle\mathcal{J}\rangle\rangle$ and hence must intersect $\mathcal{E}$. Therefore, as $\nabla^{a} r$ is not past-directed or ingoing on $J^{+}\left(\mathcal{J}^{-}\right)$by lemma $2, r$ is decreasing to the future on $\nu$ and hence $r(p) \leq r(\nu \cap \mathcal{E}) \leq \sup _{\mathcal{E}}(r)$.

To prove (b), fix any point $p \in B \cap J^{+}\left(\Sigma^{\prime}\right)$. If $r(p)$ is zero, then Eq. (4.6) is immediate. Otherwise, consider the (unique) radial ingoing and future-directed null geodesic $\nu$ with past endpoint $q$ on $\Sigma^{\prime}$ and future endpoint $p$. Denote by $\lambda$ and $l^{a}$ the parameter and associated tangent vector, respectively, in an affine parameterization of $\nu$. Then, $d r / d \lambda=l^{a} \nabla_{a} r \leq 0$ at $q$ as $\nabla^{a} r$ is outgoing at $q$. Further, along $\nu, d r / d \lambda$ remains negative as can be seen as follows. Consider,

$$
\frac{d^{2} r}{d \lambda^{2}}=l^{a} \nabla_{a}\left(l^{b} \nabla_{b} r\right)=l^{a} l^{b} \nabla_{a} \nabla_{b} r \leq 0 .
$$

The second equality follows from the fact that $\nu$ is geodetic. The inequality follows from Eq. (4.2), the fact that $l^{a}$ is null, that $\epsilon^{a}{ }_{b} l^{b}= \pm l^{a}$ (sign depending on one's choice of $\left.\epsilon_{a b}\right)$, and the fact that $\tau_{a b}$ satisfies the null-convergence condition. [Note that Eq. (4.7) is the Raychaudhuri equation for a congruence of radial null geodesics where their expansion $\theta$ is given by $\theta=\frac{2}{r} \frac{d r}{d \lambda}\left[3, A^{2}\right.$.] Therefore, $r$ is non-increasing to the future along $\nu$.

Should $\nu$ intersect the event horizon, then $r(p)$ is bounded above by $r(\nu \cap \mathcal{E})$ which in turn is bounded above by $\sup _{\mathcal{E}}(r)$. Otherwise, $\nu$ has its past endpoint on $\Sigma^{\prime} \cap B$, so as $\nabla^{a} r$ is outgoing on $\Sigma^{\prime}, r$ on $\Sigma^{\prime} \cap B$ is bounded above by $r$ on its outer boundary, which gives $r(p) \leq \max _{\Sigma^{\prime} \cap B}(r)=r(\mathcal{E} \cap \Sigma) \leq \sup _{\mathcal{E}}(r)$.

Finally, to prove (c), we note that $\Sigma^{\prime}=\Sigma$ and so by part (b), Eq. 4.6) holds on $B \cap J^{+}(\Sigma)$. To establish this bound on $B \cap J^{-}(\Sigma)$, fix any point $p$ therein. The proof proceeds identically to the above except now $\nu$ is a pastdirected ingoing radial null geodesic with past endpoint $p$ and future endpoint $q$ on $\Sigma$. [Note that $q \in B$ since $p \in B$ and $q \in J^{+}(p)$.] This time $r$ is non-increasing to the past along $\nu$ giving us the bounds $r(p) \leq r(q) \leq$ $\sup _{B \cap \Sigma}(r)=r(\mathcal{E} \cap \Sigma) \leq \sup _{\mathcal{E}}(r)$.

Lemma 7 . Fix an asymptotically flat spherically symmetric spacetime that possesses $\mathbb{R}^{3}$ Cauchy surfaces and that satisfies the dominant-energy and non-negativepressures condition [18]. Then for any spherically symmetric Cauchy surface $\Sigma$ therein, on the black-hole region $B$ with event horizon $\mathcal{E}=\partial B$, we have

$$
r \leq \max \left(\sup _{\mathcal{E}}(r), \max _{(\Sigma \cap B) \cup P}(2 m)\right)
$$

where $P$ is the subset of $\Sigma$ on which $\nabla^{a} r$ is past-directed timelike, past-directed null, or zero.

Proof. We first establish Eq. (4.8) for the portion of the black hole lying on or to the future of $\Sigma$. For this region, 
the surface $\Upsilon$ defined as the union of $\Sigma \cap B$ together with $D^{+}(\Sigma) \cap \mathcal{E}$, acts as a Cauchy surface for $D^{+}(\Sigma) \cap B$ as any past-directed causal curve with future endpoint $p \in D^{+}(\Sigma) \cap B$ and no past endpoint must intersect $\Upsilon$. To see this, for any such point $p$, consider any past-directed causal curve $\lambda$ with future endpoint $p$ and without past endpoint. The curve $\lambda$ must intersect $\Sigma$ at some point $q$ as $p \in D^{+}(\Sigma)$. If $q \in B$, then $q \in \Sigma \cap B$. If $q \notin B$, then there must a point on $\lambda$ between $p$ and $q$ that lies on $\mathcal{E}$ and thus $\lambda$ intersects $D^{+}(\Sigma) \cap \mathcal{E}$. Therefore, appealing to lemma 3 of Ref. [26] (or more correctly just repeating the argument used therein as our region is not quite a spacetime in that it has boundaries and further noting that the dominant-energy requirement was superfluous), on $B$ we have

$$
r \leq \max \left(\sup _{\Upsilon}(r), \sup _{\Upsilon}(2 m)\right) .
$$

However, we have

$$
\begin{aligned}
\max _{\Sigma \cap B}(r) & \leq \max \left(\sup _{\mathcal{E}}(r), \max _{\Sigma \cap B}(2 m)\right), \\
\sup _{D^{+}(\Sigma) \cap \mathcal{E}}(2 m) & \leq \max \left(\sup _{\mathcal{E}}(r), \max _{P}(2 m)\right) .
\end{aligned}
$$

Equation (4.10a) follows from lemma $\mathrm{C} 2$ in Appendix $\mathrm{C}$ by taking $C=\Sigma \cap B$ and noting that the boundary of $C$ is a subset of $\mathcal{E}$. Equation $4.10 \mathrm{~b}$ follows from lemma 4 . Combining these results, Eq. (4.8) follows. (Note: It is in the establishment of Eq. (4.10a) that our restriction to $\mathbb{R}^{3}$ Cauchy surfaces is used. Had $\mathbb{R} \times S^{2}$ Cauchy surfaces been allowed, $C$ would not have been compact and $r$ may be unbounded on $\Sigma \cap B$ as it is in the maximally extended Schwarzschild spacetime.)

We now establish Eq. (4.8) for the portion of the black hole lying on or to the past of $\Sigma$. For this region, the surface $\Sigma \cap B$ acts as a Cauchy surface for $D^{-}(\Sigma) \cap B$ in the sense that any future-directed causal curve with past endpoint $p \in D^{-}(\Sigma) \cap B$ and no future endpoint must intersect $\Sigma \cap B$. (Any such curve $\lambda$ must intersect $\Sigma$ at some point $q$ as $p \in D^{-}(\Sigma)$. However, $q \in B$ as $p \in B$ and hence $\lambda$ intersects $\Sigma \cap B$.) The proof now follows as before with $\Upsilon=\Sigma \cap B$.

\section{Completing the proofs of theorems 1 and 2}

To complete the proofs of theorem 1 and 2, we simply observe that theorem 1 follows from lemmas 1,5 , and 6 , while theorem 2 follows from lemmas 1,5 , and 7 .

\section{WHAT CAN BE DONE?}

A massive spherical shell surrounds a spherical vacuum (Schwarzschild) black hole so that $m=M_{1}$ inside of the shell and $m=M_{2} \gg M_{1}$ outside of the shell. An observer begins his journey on a future marginally outer trapped sphere of symmetry $\mathcal{S}$ (which will be on the event horizon if the shell of matter does not fall into the black hole). If the shell does not collapse becoming trapped within the black-hole region, then theorem 1 [part (c)] guarantees that the lifetime of our traveler is bounded above by $20 M_{1}$, which is on the order of the true maximum lifetime $\pi M_{1}$. However, suppose that the shell does fall inward creating a black hole of final mass $M_{2}$. In this case, theorem 1 bounds the lifetime of the observer by $20 M_{2}$, which is much larger than the vacuum maximum of $\pi M_{1}$. Can the observer truly live this long?

More generally, does the fact that the bound in theorem 1 is a multiple of the future asymptotic size of the black hole indicate that one can increase the lifetime of an observer therein by throwing additional matter into the hole to make it larger? Further, as it appears that the only natural upper bound for the size of the black hole $M_{\text {irr }}$ is the mass of the spacetime $M_{\mathrm{ADM}}$ (lemma A1), which corresponds to throwing all the available matter into the black hole (not that this is always possible), might one be able to increase the lifetime of our traveler within the black hole to be on the order of $M_{\mathrm{ADM}}$ ? That this is not possible in our scenario is a consequence of the following theorem.

Theorem 4. Fix a globally hyperbolic spherically symmetric spacetime satisfying the dominant-energy and non-negative-pressures conditions [18 and possessing a spherically symmetric Cauchy surface that is geodesically complete. Then, for any sphere of symmetry $\mathcal{S}$ that is future trapped, the lengths of all causal curves in $J^{+}(\mathcal{S})$ are bounded above by $10 r(\mathcal{S}) \leq 20 m(\mathcal{S})$.

Proof. This theorem follows from lemma 1 and the fact that $r \leq r(\mathcal{S})$ on $J^{+}(\mathcal{S})$, which we now establish. Fix any point $p \in I^{+}(\mathcal{S})$ with $r(p)>0$ and fix any maximal length timelike curve $\gamma$ from $\mathcal{S}$ to $p$. The curve $\gamma$ is radial and geodetic with $r$ positive thereon. It follows from the non-negative-pressures condition that $d^{2} r / d t^{2} \leq-m / r^{2}$ on $\gamma$ (where $t$ is the proper time along $\gamma$ ), and since $m$ is non-negative by lemma $5, d^{2} r / d t^{2} \leq 0$ on $\gamma$. However, as $\nabla^{a} r$ is future-directed timelike, future-directed null, or zero on $\mathcal{S}, d r / d t$ must be nonpositive on $\mathcal{S}$. Therefore, $d r / d t \leq 0$ on all of $\gamma$. Thus, $r(p) \leq r(\mathcal{S})$. That $r \leq r(\mathcal{S})$ on the boundary of $J^{+}(\mathcal{S})$ follows by continuity of $r$ (or by a slight modification of the above argument for the null case).

This theorem bounds the length of the journey of the observer in the scenario above by $10 r(\mathcal{S}) \leq 20 m(\mathcal{S})=$ $20 M_{1}$, which shows that the additional matter cannot increase his lifetime to be on the order of $M_{2}$. In fact, it turns out that in this case (and those like it) the additional matter never increases the lifetime of an observer within. (See theorem 6 below.)

To address the more general problem of whether we can increase the lifetime of an observer within a black hole, some care is needed as the problem is subtle for at least three reasons. First, while it may be possible to increase the amount of time an observer can possibly exist within a black hole, it may just be that none of this increase is 
available to the observer that is already within the blackhole region. That is, while we expect that observers can exist longer in larger black holes (as is certainly true for vacuum Schwarzschild black holes), and it is true that by throwing in matter we can increase the amount of time an observer has spent within a black hole (by making the black hole larger, which is related to our next point), it may be that an observer still cannot live any longer than he would have otherwise. Second, the very notion of a black hole is a nonlocal concept requiring a knowledge of the entire future history for its determination. For example, while the spacetime corresponding to a static spherically symmetric shell of matter with a flat interior has no black-hole region, if we choose to "turn off" the pressure that keeps the shell from collapsing inwards, the new spacetime will possess a nonempty black-hole region. So, while under one evolution an observer may begin his journey outside of the black-hole region, in another he may already be within this region. Third, the manipulation of matter is a delicate issue in general relativity. We cannot move matter around as we like, e.g., pulling two stars together or pushing them apart. The pushing and pulling involve stresses that must be taken into account as demanded by Einstein's theory of gravity.

To take care of these subtleties, we formulate our problem as an initial-value problem as follows. Recall that an initial data set $I$ for general relativity consists of a triple $I=\left(\Sigma, q_{a b}, K_{a b}\right)$ 叫. Here $\Sigma$ is a three-manifold (physically the spacetime at an "instant"), $q_{a b}$ is a Riemannian metric on $\Sigma$ (physically the metric induced from the spacetime metric $g_{a b}$ ), and $K_{a b}$ is a symmetric tensor on $\Sigma$ (physically the extrinsic curvature of $\Sigma$ in $M$ ). On $\Sigma, q_{a b}$ and $K_{a b}$ are not freely specifiable. Instead, the initial-value constraint equations of general relativity impose a relation between these quantities and the energy density and mass-current density of the matter fields. (See Eqs. (10.2.41) and (10.2.42) of Ref. [4].)

Fix an initial data set $I$ and a point $p$ in the threemanifold $\Sigma$ associated with $I$. Our knowledge of $I$ corresponds to a knowledge of the state of the Universe at an "instant of time", while our knowledge of $p$ corresponds to our knowledge of the position of our observer at that time. If we had a detailed description of the matter fields (in particular their initial data satisfying the constraint equations and the equations governing their evolution) then with Einstein's equation we could evolve this initial data to produce a unique spacetime (up to diffeomorphisms). However, we wish investigate the behavior of gravitational fields without this detailed knowledge of the matter fields, One way around this problem is to restrict ourselves to vacuum spacetimes. Instead, we choose to proceed as follows.

To restrict ourselves to spacetimes that are "physical" in the sense that their matter content could be considered in some sense "ordinary", we shall restrict ourselves to spacetimes whose Einstein tensor satisfies a fixed set of inequalities (energy conditions). For definiteness, here we shall demand that the spacetime satisfy the dominant- energy and non-negative-pressures conditions [18]. We shall call a spacetime $\left(M, g_{a b}\right)$ satisfying these energy conditions a possible evolution of an initial data set $I$ if there is a Cauchy surface $S$ in $M$ such that the induced data on $S$ coincides with that of $I$. Note that without the energy conditions, there will be many possible evolutions of any given initial data set. In fact, we can "evolve" any initial data set $I_{1}$ to any other initial data set $I_{2}$ in the sense that there will be a single spacetime that is a possible evolution of both $I_{1}$ and $I_{2}$. (In the terminology of Geroch [44], without restrictions, we can build any initial data set from any other initial data set.) It is worth noting that when we restrict ourselves to spacetimes satisfying the dominant-energy condition, if the initial data is vacuum in the sense that the associated energy and mass-current densities (which can be calculated using the initial-value constraint equations of general relativity), then a possible evolution of $I$ is everywhere vacuum and therefore is unique (up to diffeomorphisms). More generally, however, there will be many possible evolutions of a given initial data set.

From a possible evolution of $I$, we can calculate the least upper bound to the lengths of causal curves having past endpoint $p$. By looking at this least upper bound over the set of all possible evolutions, we can find which possible evolution gives our observer the longest possible lifetime. If $p$ is not within the black hole for every possible evolution of $I$, then there is no finite upper bound on the lifetime of our observer as there will be a possible evolution for which there is a causal curve with past endpoint $p$ and future endpoint on $\mathcal{J}^{+}$(with such a curve having infinite length). In other words, in this case we can choose our possible evolution so that the observer in fact was never within a black hole. However, if $p$ is in the subset $\mathcal{B}$ of $\Sigma$ consisting of all the points of $\Sigma$ that lie within the black-hole region of each possible evolution, then an observer that begins a journey at $p \in \mathcal{B}$ is guaranteed to lie within the black-hole region independent of which possible evolution of $I$ we choose. Note that $\mathcal{B}$ is a closed subset of $\Sigma$ (being the intersection of a collection of closed sets), and that when there is only a single possible evolution (e.g., a vacuum spacetime), then $\mathcal{B}=B \cap \Sigma$. Of course, $\mathcal{B}$ may be empty. With this definition, a question relevant to our original problem is the following.

Question 1. Given an initial data set $I$, what is the least upper bound on the lengths of causal curves in $J^{+}(\mathcal{B})$ over the set of all possible evolutions of $I$ ?

Physically, this upper bound corresponds to the greatest lifetime an observer, beginning his journey at some point in $\mathcal{B}$, can hope to achieve by our (allowed) manipulations of the matter in the spacetime. Unfortunately, this question appears to be as difficult to answer as proving conjectures 1 and 2. Even the task of computing $\mathcal{B}$ from $I$ appears difficult as finding all possible evolutions and their associated black-hole regions is nontrivial. Are there any sufficient conditions for knowing whether a given point $p \in \Sigma$ is in $\mathcal{B}$ ? It is well known that future 
trapped surfaces must lie within the black-hole region of an asymptotically predictable spacetime satisfying the null-convergence condition. Further, the total trapped region $\mathcal{T}$ associated with an initial data surface (or initial data set) shares this property [4]. Therefore, $\mathcal{T} \subset \mathcal{B}$. In other words, if an observer lies in the total trapped region, it is assured that there is no possible evolution that will allow him to escape to infinity. So, restricting ourselves to observers that begin their journey from some point in $\mathcal{T}$, we are led to the following question.

Question 2. Given an initial data set $I$, what is the least upper bound on the lengths of causal curves in $J^{+}(\mathcal{T})$ over the set of all possible evolutions of $I$ ?

This question also appears quite difficult to answer. However, restricting ourselves to spherically symmetric initial data sets and spherically symmetric possible evolutions (spherically symmetric spacetimes), a partial answer to this last question is given by the following theorem.

Theorem 5. Fix a spherically symmetric initial data set $I$ with three-manifold $\Sigma \approx \mathbb{R}^{3}$. Denoting the total trapped region in $\Sigma$ by $\mathcal{T}$, the lengths of all causal curves in $J^{+}(\mathcal{T})$ are bounded above by $20 \max _{\mathcal{T}}(m)$ for any possible evolution satisfying the dominant-energy and nonnegative-pressures conditions [18].

Proof. This theorem follows from lemma 1 and the fact that $r \leq \max _{\mathcal{T}}(2 m)$ on $J^{+}(\mathcal{T})$, which we now establish. As in the proof of lemma 7, we begin by noting that the boundary of $J^{+}(\mathcal{T})$ acts much like a Cauchy surface for $J^{+}(\mathcal{T})$. On $\left(\partial J^{+}(\mathcal{T})\right) \backslash \mathcal{T}$, denote a null generator of this null boundary by $\nu$, affinely parameterized by $\lambda$. Then, by the null-convergence condition (which follows from both the dominant-energy and non-negativepressures conditions), we have $d^{2} r / d \lambda^{2} \leq 0$. Therefore, as $d r / d \lambda=0$ on the apparent horizon $\mathcal{A}=\partial \mathcal{T}, d r / d \lambda \leq$ 0 all along $\nu$ to the future of $\mathcal{A}$, and so $r \leq r(\mathcal{A})$ thereon as well. Further, by lemma $\mathrm{C} 2, \max _{\mathcal{T}}(r) \leq \max _{\mathcal{T}}(2 m)$, where we have used the fact that $r(\mathcal{A})=2 m(\mathcal{A})$. Repeating the argument of lemma 3 in Ref. [26] [and noting that $\nabla^{a} r$ cannot be past-directed timelike on $\left.\left(\partial J^{+}(\mathcal{T})\right) \backslash \mathcal{T}\right]$, we have $r \leq \max _{\mathcal{T}}(2 m)$ on $J^{+}(\mathcal{T})$.

Therefore, we have a bound on the lifetime of observers beginning their journey in the total trapped region of a Cauchy surface in terms of the initial data on that region. In particular, this bound is insensitive to the final asymptotic size of the black hole (unlike the bounds given by theorems 1 and 2).

So, can we increase the lifetime of an observer in a black hole by manipulating the matter? In general, yes, since not all possible evolutions allow for equally long causal curves. Can we increase it to be on the order of $M_{\mathrm{ADM}}$ ? While this remains unanswered, for certain observers in the spherically symmetric case, theorems 4 and 5 show that this is not always possible. Are there any initial data sets where adding matter cannot lengthen the lifetime of an observer in the black hole at all? Yes. We state, without proof, the following theorem giving a class of initial data (a vacuum Schwarzschild black hole of mass
$M$ surrounded by matter) showing that the lifetime of observers in the Schwarzschild region cannot be increased beyond the vacuum maximum $\pi M$.

Theorem 6. Fix a spherically symmetric initial data set $I$ such that: (1) The data on its total trapped region $\mathcal{T}$ is isometric to the data induced on a Cauchy surface in maximally extended Schwarzschild spacetime of positive mass $M$ with the region corresponding to $\mathcal{T}$ lying in region II of Fig. 11 (i.e., $\nabla^{a} r$ is future-directed timelike everywhere inside of $\mathcal{T}$ ); (2) Outside of $\mathcal{T}, \nabla^{a} r$ is outgoing spacelike. Restricting ourselves to spacetimes satisfying the dominant-energy and non-negative-pressures conditions 18], the lifetime of an observer to the future of some point $p \in \mathcal{T}$ under a spherically symmetric possible evolution of $I$ is no greater than the maximum lifetime under a spherically symmetric possible evolution with $J^{+}(\mathcal{T})$ being vacuum, which is no greater than $\pi M$. More weakly, the least upper bound to the lengths of causal curves in $J^{+}(\mathcal{T})$ over the set of spherically symmetric possible evolutions is $\pi M$.

\section{DISCUSSION}

As the proofs of conjectures 1 and 2 in the spherically symmetric case involve a detailed use of the fields $r$ and $m$, concepts for which we do not have an adequate generalization (at least none that are obviously useful for our purposes), it would seem that their proof in the general case will be a difficult task. However, we do have a few hints as to how one might proceed.

One possible step towards a proof of conjecture 1 is provided by generalizing the lemma bounding the size of the spheres of symmetry $r$ in the black hole region of a spherically symmetric spacetime (lemma 6) along the following lines. Consider a "cut" $K$ of past null infinity $\mathcal{J}^{-}$and consider the null boundary $\partial J^{+}(K)$. The null generators of this boundary are past complete and have positive expansion thereon (to the past). Therefore, the area of a cross-section of $\partial J^{+}(K)$ will be decreasing to the future and, therefore, the areas of cross-sections of $\partial J^{+}(K)$ in the black-hole region will be bounded by the area associated with a cross-section of the event horizon, which is bounded by the future asymptotic area of the black hole's event horizon. (This is simply a mimicry of the proof used in lemma 6.) Even with such a result, this is still far from showing that there are no long causal curves in the portion of the black hole region lying to the future of $\mathcal{J}^{-}$.

Perhaps a good place to start in the proof of conjecture 2 would be to attempt a proof of a generalized version of theorem 5, i.e., drop the spherically symmetry requirement and replace $\max _{\mathcal{T}}(m)$ by some more general quantity $M_{\mathcal{T}}$ constructed on $\mathcal{T}$. Although such a theorem would not place an upper bound on the lifetimes of all observers in a black hole region, it would bound those lying to the future of a total trapped region (which is a 
subset of the total black hole region). Further, such a theorem would be a very nice strengthening of Penrose's 1965 theorem that shows that $J^{+}(\mathcal{T})$ cannot be future null complete 11.

We end with a few remarks and questions for consideration. (1) Do conjectures 1 and 2 in fact hold with $k=2 \pi$ (the smallest possible value for which they can hold as shown by our analysis of the Kerr and dust-ball spacetimes)? (2) Can weaker versions of conjectures 1 and 2 be proven? For instance, a theorem that merely asserts that the lifetimes of observers in the black-hole region will be bounded, or merely that the lifetimes must be finite (no global upper bound), would be of interest. (3) If conjecture 1 or 2 is false, does a counterexample to either conjecture exist in the literature, or are the spacetimes providing counterexamples peculiar in some way so that they have been missed? (4) Can the energy conditions in theorems 1 and 2 be weakened? In particular, it would be nice if the non-negative-pressures condition could be replaced by a weaker condition stating that the pressure is not "too negative" compared to the energy density. (5) As has been mentioned, Theorems 1 and 4 are slightly awkward in that they require the existence of a geodesically complete spherically symmetric Cauchy surface so that $m$ will be non-negative everywhere as needed by lemma 1. Can the requirement that $m$ be non-negative in lemma 1 be dropped, thereby allowing for improved versions of theorems 1 and 4 ?

\section{ACKNOWLEDGMENTS}

I would like to thank Robert Geroch for initially directing my attention to the possibility of proving a theorem such as theorems 1 and 2 and for the discussions with both him and Robert Wald regarding this work.

\section{APPENDIX A: BOUNDING THE AREA OF A SPHERICALLY SYMMETRIC BLACK HOLE}

Theorem A1. For a globally hyperbolic asymptotically flat spherically symmetric spacetime satisfying the dominant-energy condition 18 and with $\mathcal{J}^{+}$future complete, the area $A$ of the event horizon is always bounded above by

$$
A \leq 16 \pi M_{\mathrm{ADM}}^{2} .
$$

Equivalently, on the event horizon: $M_{\mathrm{irr}} \leq M_{\mathrm{ADM}}$; and $r \leq 2 M_{\mathrm{ADM}}$.

Proof. The basic idea behind the proof is to construct a "retarded-time function" $u$ such that $u \rightarrow \infty$ as we approach the event horizon of the black hole provided that $\mathcal{J}^{+}$is complete and then show that this is not this case if $r$ is anywhere larger than $2 M_{\mathrm{ADM}}$ on the event horizon. Essentially, the proof uses the outgoing EddingtonFinkelstein coordinates ( $r$ and $u$, together with two angular coordinates [34]). However, we choose not to actually "work in these coordinates" as it is easier to just treat $r$ and $u$ as fields and work with their properties.

We begin by constructing a "retarded-time function" $u$ on $J^{-}\left(\mathcal{J}^{+}\right)$as follows. Through each point $p$ in the spacetime with $r(p)>0$, there exists two spherically symmetric null surfaces containing $p$. In the region outside of the black hole, i.e., $J^{-}\left(\mathcal{J}^{+}\right)$, we shall "label" the outgoing surfaces (by which is meant those whose tangent vectors can be taken to be outgoing and future-directed) by defining the scalar field $u$ to be constant on these null surfaces and requiring that $\nabla^{a} u \nabla_{a} r \rightarrow-1$ as we approach $\mathcal{J}^{+}$. This choice uniquely defines $u$ everywhere on $J^{-}\left(\mathcal{J}^{+}\right)$(up to the addition of an overall constant to $u$ ) and has the advantage that the completeness of $\mathcal{J}^{+}$is then equivalent to the property of $u$ assuming all values on $J^{-}\left(\mathcal{J}^{+}\right)$.

Set $k^{a}=-\nabla^{a} u$. Then $k^{a}$ is radial, outgoing, futuredirected, null, tangent to surfaces of constant $u$, and geodetic (as is any null vector field arising from a gradient). Further, on $J^{-}\left(\mathcal{J}^{+}\right)$(where $r>0$ ), define $l^{a}$ to be the radial outgoing past-directed null vector field such that $k^{a} l_{a}=+2$. Then, setting $r^{\prime}=k^{a} \nabla_{a} r$, we have

$$
\begin{aligned}
& l^{a} \nabla_{a} u=-2, \\
& l^{a} \nabla_{a} r=\frac{1}{r^{\prime}}(1-2 m / r) .
\end{aligned}
$$

Equation (A2a) follows from the definition of $k^{a}$ and our normalization requirement for $l^{a}$. Equation (A2b follows from the fact that $h^{a b}=k^{(a} l^{b)}$ so that $(1-2 m / r)=$ $\nabla^{a} r \nabla_{a} r=\left(k^{a} \nabla_{a} r\right)\left(l^{a} \nabla_{a} r\right)$. [Note that $r^{\prime}(p)$ is positive for all $p \in J^{-}\left(\mathcal{J}^{+}\right)$, as otherwise the sphere of symmetry containing $p$ would be an outer trapped surface. Such surfaces cannot occur in this region provided the null-convergence condition holds, as it does in our case. Indeed, by the null-convergence condition, $r^{\prime \prime} \leq 0$, and since $r^{\prime}$ is unity at $\mathcal{J}^{+}$, we have $r^{\prime} \geq 1$ everywhere on $J^{-}\left(\mathcal{J}^{+}\right)$.]

Now suppose that for some sphere of symmetry $\mathcal{S}$ on the event horizon $r(\mathcal{S})>2 M_{\mathrm{ADM}}$. Setting $\Lambda=\langle\langle\mathcal{J}\rangle\rangle \cap$ $\left(J^{-}(\mathcal{S})\right)^{c}$, then $r>r(\mathcal{S})$ everywhere on $\Lambda$. (See Fig. 5.) To see this, fix any point $p \in \Lambda$ and let $\lambda$ denote the spherically symmetric ingoing null surface containing $p$. Similarly, let $\sigma$ be the spherically symmetric ingoing null surface containing $\mathcal{S}$. Since $r$ and $u$ are continuous, for any number $\epsilon>0$ we can find a constant $u_{0}$ such that $r(s)>r(\mathcal{S})-\epsilon$ for all $s \in \sigma$ with $u(s)>u_{0}$. Let $\nu$ be the maximal integral curve of $k^{a}$ containing $s$. Then, setting $q=\nu \cap \lambda$, it follows from the fact that $\nabla^{a} r$ is outgoing or past-directed (yet not parallel to $\left.k^{a}\right)$ on $J^{-}\left(\mathcal{J}^{+}\right)$that $r(q)>r(s)$. Further, choosing $s$ so that $u(s)=u(q)>$ $u(p)$ (i.e., so that $q$ lies to the future of $p$ ) we have $r(p)>$ $r(q)$. Putting this all together, we have, $r(p)>r(q)>$ $r(s)>r(\mathcal{S})-\epsilon$ for all $\epsilon>0$ and so $r>r(\mathcal{S})$ on $\Lambda$. (That $r \neq r(\mathcal{S})$ on $\Lambda$ follows from the fact that $\nabla^{a} r$ is outgoing spacelike on the open set $\Lambda$.) 


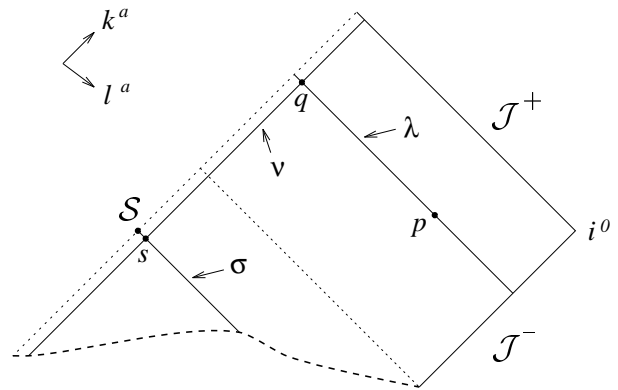

FIG. 5. The construction performed on $J^{-}\left(\mathcal{J}^{+}\right)$used to show that $r>r(\mathcal{S})$ on $\Lambda=\langle\langle\mathcal{J}\rangle\rangle \cap\left(J^{-}(\mathcal{S})\right)^{c}$. (In the case depicted here, $\Lambda=\langle\langle\mathcal{J}\rangle\rangle$.) For $p \in \Lambda$, we have $r(p)>r(q)>r(s)>r(\mathcal{S})-\epsilon$. Therefore, as $r(p)>r(\mathcal{S})-\epsilon$ for all $\epsilon>0$, we have $r(p)>r(\mathcal{S})$. (Equality cannot be attained as $\nabla^{a} r$ is outgoing spacelike on the open set $\Lambda$.) The dotted line denotes where the event horizon would be if it were "all there". In that case, the construction can be simplified by taking $\nu$ to lie on the horizon, thereby avoiding the construction of $\sigma$ and the need to take a limit as $\epsilon \rightarrow 0$.

Further, on $\Lambda$, we have

$$
l^{a} \nabla_{a} r \geq 1-2 M_{\mathrm{ADM}} / r .
$$

To see this, consider how $l^{a} \nabla_{a} r$ changes along an integral curve of $k^{a}$ parameterized by $r$,

$$
\begin{aligned}
\frac{d}{d r}\left(l^{a} \nabla_{a} r\right) & =\frac{1}{r^{\prime}} k^{b} \nabla_{b}\left(l^{a} \nabla_{a} r\right) \\
& =\frac{1}{r^{\prime}} k^{b} l^{a} \nabla_{b} \nabla_{a} r \\
& \leq \frac{1}{r^{\prime}} \frac{2 m}{r^{2}} .
\end{aligned}
$$

The first equality follows from the definition of $r^{\prime}$, the second from the fact that $k^{b} \nabla_{b} l^{a}=0$, and the third follows from Eq. (4.3) and the dominant-energy condition. Therefore, dividing Eq. (A4c) by Eq. (A2b) and noting that $l^{a} \nabla_{a} r$ is positive on $\Lambda$ (as it is a subset of $\langle\langle\mathcal{J}\rangle\rangle$ ), we have

$$
\left(l^{a} \nabla_{a} r\right)^{-1} \frac{d}{d r}\left(l^{a} \nabla_{a} r\right) \leq \frac{2 m}{r^{2}}(1-2 m / r)^{-1} .
$$

Using the facts that $r>2 M_{\mathrm{ADM}} \geq 2 m$ on $\Lambda$, we find

$$
\left(l^{a} \nabla_{a} r\right)^{-1} \frac{d}{d r}\left(l^{a} \nabla_{a} r\right) \leq \frac{2 M_{\mathrm{ADM}}}{r^{2}}\left(1-2 M_{\mathrm{ADM}} / r\right)^{-1} .
$$

Integrating this and using the fact that $l^{a} \nabla_{a} r \rightarrow 1$ as $r \rightarrow \infty$ along the integral curve of $k^{a}$ [by Eq. (A2b) and the fact that $m \leq M_{\mathrm{ADM}}$ on $\left.\langle\langle\mathcal{J}\rangle\rangle\right]$, gives Eq. (A3).

We can now establish an upper bound on $u$ as follows. Let $\lambda$ be a maximal integral curve of $l^{a}$ in $\Lambda$. Parameterizing this curve by $u$, we have

$$
-\frac{d r}{d u}=\frac{\left(l^{a} \nabla_{a} r\right)}{\left(-l^{a} \nabla_{a} u\right)} \geq \frac{1}{2}\left(1-2 M_{\mathrm{ADM}} / r\right) .
$$

Setting $r^{*}=r+2 M_{\mathrm{ADM}} \ln \left(r-2 M_{\mathrm{ADM}}\right)$ and integrating Eq. (A7) along the curve $\lambda$ from a point $p$ to a point $q$ lying to the future of $p$, we have

$$
u(q) \leq u(p)+2\left(r^{*}(p)-r^{*}(q)\right) .
$$

Since $r>r(\mathcal{S})$ on $\Lambda, r^{*}$ is bounded from below by $r^{*}(\mathcal{S})$ on $\Lambda$, which is finite as $r(\mathcal{S})>2 M_{\mathrm{ADM}}$. Therefore, Eq. (A8) gives an upper bound on allowed values of $u$ contradicting the completeness of $\mathcal{J}^{+}$. So, on the event horizon, $r$ can be no greater than $2 M_{\mathrm{ADM}}$.

Theorem A2. Fix a globally hyperbolic asymptotically flat spherically symmetric spacetime satisfying the dominant-energy condition [18] with $\mathcal{J}^{+}$future complete and the event horizon complete in the sense that a future incomplete timelike or null geodesic in $\langle\langle\mathcal{J}\rangle\rangle$ can be assigned an endpoint on the event horizon (in other words, the event horizon is "all there") and the null geodesic generators of the event horizon are future complete. Then, $r / 2$ and $m$ have the same limit to the future on the event horizon. (In other words, the irreducible mass $M_{\text {irr }}$ and mass $m$ have the same future asymptotic limits to the future on the event horizon.)

Proof. Denote by $\lambda$ and $k^{a}$ the parameter and associated tangent vector, respectively, in an affine parameterization of a null geodesic generator $\nu$ or the event horizon. Further, let $l^{a}$ denote the radial null vector parallel transported along $\nu$ with $k^{a} l_{a}=+2$ thereon. Denote the future asymptotic value of $m$ on the event horizon by $M_{f}$. If $r \leq 2 M_{f}$ on all of $\nu$, then since $2 m \leq r$ on the portion of $\nu$ lying to the future of $\mathcal{J}^{-}, r$ must have $2 M_{f}$ as a future limit. So, suppose that at some point on $\nu$ that $r>2 M_{f}$. Since $r$ is non-decreasing to the future on $\nu$, this inequality will continue to hold. Then, repeating the argument that led to Eq. (A5), this equation again holds with our $l^{a}$ on the portion of $\nu$ lying to the future of $\mathcal{J}^{-}$. As $m \leq M_{f}$ on this portion, we have

$$
\left(l^{a} \nabla_{a} r\right)^{-1} \frac{d}{d r}\left(l^{a} \nabla_{a} r\right) \leq \frac{2 M_{f}}{r^{2}}\left(1-2 M_{f} / r\right)^{-1} .
$$

Integrating this we have

$$
l^{a} \nabla_{a} r \leq C\left(1-2 M_{f} / r\right),
$$

for some positive constant $C$. Noting that $h^{a b}=k^{(a} l^{b)}$ on $\nu$, so that $\left(k^{a} \nabla_{a} r\right)\left(l^{a} \nabla_{a} r\right)=1-2 m / r$, we have

$$
\frac{d r}{d \lambda}=k^{a} \nabla_{a} r=\frac{1}{\left(l^{a} \nabla_{a} r\right)}(1-2 m / r) \geq \frac{1}{C} .
$$

Integrating this, we see that $r \rightarrow \infty$ as $\lambda \rightarrow \infty$. However, this contradicts the fact that $r$ is bounded by $2 M_{\mathrm{ADM}}$ on the event horizon by theorem A1. Therefore, the event horizon cannot be complete without $r$ and $2 m$ having the same future limits. 


\section{APPENDIX B: THE CONSTRUCTION OF DUST-BALL SPACETIMES}

In some detail, the construction of a dust-ball spacetime proceeds as follows.

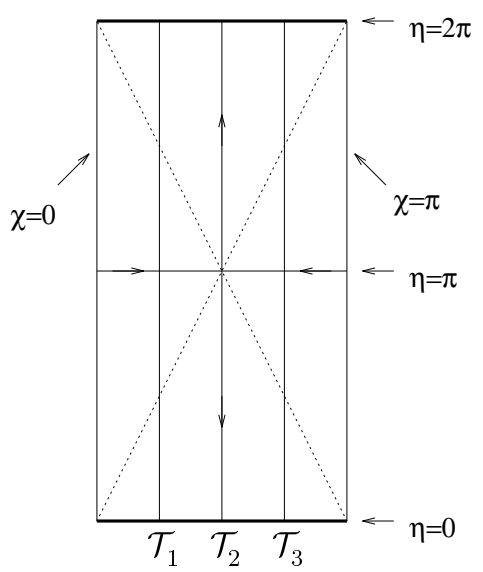

FIG. 6. A spacetime diagram representing a $k_{\mathrm{RW}}=+1$ Robertson-Walker spacetime with dust as a source. In this diagram, null rays perpendicular to the spheres of symmetry have slopes of \pm 1 . Surfaces of constant $\eta$ are surfaces of homogeneity with $\eta=\pi$ being the maximal hypersurface. The two diagonal timelike surfaces $(\eta=2 \chi$ and $\eta=2 \pi-2 \chi)$ are where $r=2 m$ (and $r \neq 0$ ). These surfaces divide the spacetime into four regions each on which $\nabla^{a} r$ points into a different quadrant. The timelike surfaces $\mathcal{T}_{1}, \mathcal{T}_{2}$, and $\mathcal{T}_{3}$ are surfaces of constant $\chi$ and are generated by the (geodetic) flow of the dust (i.e., the integral curves of the flow are tangent to these surfaces). On each surface, $\chi=\chi_{0}$ for some constant $\chi_{0}$. For $\mathcal{T}_{1}, 0<\chi_{0}<\pi / 2$; for $\mathcal{T}_{2}, \chi_{0}=\pi / 2$; for $\mathcal{T}_{3}$, $\pi / 2<\chi_{0}<\pi$.

The Robertson-Walker spacetimes are spherically symmetric and can be coordinated (everywhere excepting where $r=0$ ) so that the metric is given by

$$
g_{a b}=a^{2}(\eta)\left(-(d \eta)_{a}(d \eta)_{b}+(d \chi)_{a}(d \chi)_{b}+f_{k}^{2}(\chi) \Omega_{a b}\right),
$$

where $\Omega_{a b}$ is a unit-metric on the two-sphere,

$$
f_{k_{\mathrm{RW}}}(x)= \begin{cases}\sin (x) & \text { for } k_{\mathrm{RW}}=+1 \\ x & \text { for } k_{\mathrm{RW}}=0 \\ \sinh (x) & \text { for } k_{\mathrm{RW}}=-1\end{cases}
$$

and $k_{\mathrm{RW}}$ tells us which family we are considering. (The sign of $k_{\mathrm{RW}}$ gives the sign of the intrinsic Ricci scalar curvature of the surfaces of homogeneity.) Surfaces of constant $\eta$ are the surfaces of homogeneity and the integral curves of the fluid flow lie in surfaces of constant $\chi$ and are perpendicular to the spheres of symmetry. Note that the allowed range for $\chi$ in expressing the metric in this form is $\chi>0$ in the $k_{\mathrm{RW}}=-1$ and $k_{\mathrm{RW}}=0$ cases while $0<\chi<\pi$ in the $k_{\mathrm{RW}}=+1$ case.
From Eq. (B1), the size $r$ of a sphere of symmetry is given by

$$
r(\eta, \chi)=a(\eta) f_{k_{\mathrm{RW}}}(\chi) .
$$

With dust as a source, $a(\eta)$ is given by

$$
a(\eta)=C f_{k_{\mathrm{RW}}}^{2}(\eta / 2)
$$

for some constant $C$. Using Eq. (B3) and Eq. (4.2), the mass $m$ associated with each sphere of symmetry is calculated to be

$$
m(\eta, \chi)=\frac{C}{2} f_{k_{\mathrm{RW}}}^{3}(\chi) .
$$

Notice that $m$ is constant along the integral curves of the fluid flow, as it must be as dust has zero pressure (see Eq. (4.4)).

To construct a dust-ball spacetime, we fix a dust-filled Robertson-Walker spacetime as described above (i.e., fix some $k_{\mathrm{RW}}$ and $C$ ) and choose a number $\chi_{0}$ (within the allowed range). We then take the region in the RobertsonWalker spacetime with $\chi \leq \chi_{0}$ and attach its boundary $\mathcal{T}$ (being the timelike three-surface where $\chi=\chi_{0}$ ) to an appropriate similar three-surface $\mathcal{T}^{\prime}$ of an appropriate extended Schwarzschild spacetime. In order that there not be any "surface layers" where the spacetimes are glued together, it is necessary that $m$ be a continuous function on the newly created spacetime. Therefore, the mass of the appropriate Schwarzschild spacetime is simply $m$ evaluated on $\mathcal{T}$. As the ADM mass $M_{\mathrm{ADM}}$ of the spacetime so constructed is the mass of the Schwarzschild portion, we therefore have

$$
M_{\mathrm{ADM}}=\frac{C}{2} f_{k_{\mathrm{RW}}}^{3}\left(\chi_{0}\right) .
$$

We shall omit the remainder of the details of this matching except to note that, in the $k_{\mathrm{RW}}=+1$ case, when: $\chi_{0} \leq \pi / 2, \mathcal{T}^{\prime}$ lies in regions I, II, and III and thereby "covers up" all of region IV in Fig. 1; otherwise $\mathcal{T}^{\prime}$ lies in regions II, III, and IV and thereby cannot be reached from the asymptotic region (region I) without entering the black hole.

In Figs. 3 and 4 we have sketched two such dust-ball spacetimes. In Fig. 3, $\chi_{0}<\pi / 3$, while in Fig. 1, $\chi_{0}>$ $2 \pi / 3$. In each, we have divided the spacetime into a number of regions according to the following scheme. On $\mathrm{I}, \mathrm{I}^{\prime}, \mathrm{I}^{\prime \prime}$, and $\mathrm{I}^{\prime \prime \prime}, \nabla^{a} r$ is outgoing and: I lies to the future of $\mathcal{J}^{-}$and the past of $\mathcal{J}^{+}$(i.e, I is the asymptotic region); $\mathrm{I}^{\prime}$ is in the future of $\mathcal{J}^{-}$but not in the past of $\mathcal{J}^{+} ; \mathrm{I}^{\prime \prime}$ lies past of $\mathcal{J}^{+}$but not in the future of $\mathcal{I}^{-} ; \mathrm{I}^{\prime \prime \prime}$ lies neither in the future of $\mathcal{J}^{-}$nor in the past of $\mathcal{J}^{+}$. On II and $\mathrm{II}^{\prime}, \nabla^{a} r$ is future-directed and II lies to the future of $\mathcal{J}^{-}$ while II' does not. On III and III', $\nabla^{a} r$ is past-directed and III lies to the past of $\mathcal{J}^{+}$while III' does not. On IV, $\nabla^{a} r$ is ingoing. For the dust-ball spacetimes, regions I, II, and III always exist while: $\mathrm{I}^{\prime}$ and $\mathrm{I}^{\prime \prime}$ exist only for $\chi_{0}<2 \pi / 3 ; \mathrm{I}^{\prime \prime \prime}$ exists only for $\chi_{0}>\pi / 3 ; \mathrm{II}^{\prime}, \mathrm{III}^{\prime}$, and IV exist only for $\chi_{0}>\pi / 2$. 
Note that the outer surface of the dust ball crosses the event horizon of the black hole where the surface $\chi=\chi_{0}$ intersects the surface $\eta=2 \pi-2 \chi$, i.e., at $\eta=\eta_{0}=$ $2 \pi-2 \chi_{0}$. (See Figs. 6, 3, and 4.) Therefore, on the event horizon in the dust-filled portion of the spacetime we have $\eta=\eta_{0}+\left(\chi-\chi_{0}\right)=\left(2 \pi-3 \chi_{0}\right)+\chi$. In particular, in the $k_{\mathrm{RW}}=+1$ case, we see that the black hole is eternal, in the sense that every Cauchy surface will intersect the black-hole region, if $\chi_{0} \geq 2 \pi / 3$.

\section{APPENDIX C: TWO LEMMAS BOUNDING $r$}

Lemma C1. Fix globally hyperbolic spherically symmetric spacetime satisfying the non-negative-pressures condition 18]. Fix any Cauchy surface surface $\Sigma$ therein and let $P$ denote the subset of $\Sigma$ on which $\nabla^{a} r$ is pastdirected timelike, past-directed null, or zero. Then, for a point $p \in D^{+}(\Sigma)$ at which $\nabla^{a} r$ is past-directed timelike, past-directed null, or zero, we have

$$
r(p) \leq 2 m(p) \leq \sup _{P}(2 m)
$$

Proof. The first inequality is immediate from the definition of $m$ while the proof of the second is obtained by repeating the argument given in the latter portion of the proof of lemma 3 in Ref. 26] and noting that the theorem's requirement that the dominant-energy condition hold can be dropped. (That is, as the null-convergence condition follows from the non-negative-pressures condition and it can be shown that the existence of a timelike vector $t^{a}$ for which the last term in Eq. (2.16) in Ref. 26] is non-negative follows from the null-convergence condition, the requirement that the dominant-energy condition hold was superfluous.) $\square$

Lemma C2. Fix a globally hyperbolic spherically symmetric spacetime and a spherically symmetric Cauchy surface $\Sigma$ therein. For a spherically symmetric compact subset $C$ of $\Sigma$, define $\partial^{+} C$ to be those components of $\partial C$ for which $\nabla^{a} r$ is spacelike and $v^{a} \nabla_{a} r>0$ for vectors $v^{a}$ on $\partial C$ (tangent to $\Sigma$ ) and pointing out of $C$. Then, we have

$$
\max _{C}(r) \leq \max \left(\max _{\partial^{+} C}(r), \max _{C}(2 m)\right) .
$$

Proof. Let $p$ be any point where $r$ reaches its maximum value on $C$. If $\nabla^{a} r$ is non-spacelike at $p$, then $\max _{C}(r)=r(p) \leq 2 m(p) \leq \max _{C}(2 m)$, where the first inequality follows from the definition of $m$ given by Eq. (4.2). Otherwise, if $\nabla^{a} r$ is spacelike at $p$, then $p$ must be on $\partial^{+} C$ for otherwise if $p$ is in the interior of $C$ or on any other part of the boundary, there exist vectors $v^{a}$ (either in $C$ or pointing into $C$ ) such that $v^{a} \nabla_{a} r>0$ thereby violating the maximality of $r$ at $p$ on $C$. Therefore, in this case $\max _{C}(r)=r(p)=\max _{\partial+C}(r)$. Combining these two cases, Eq. (C2) follows.
[1] K. S. Thorne, Black holes and time warps: Einstein's outrageous legacy (W. W. Norton, New York, 1994).

[2] W. Israel, in Three Hundred Years of Gravitation, edited by S. W. Hawking and W. Israel (Cambridge University Press, Cambridge, England, 1989), Chap. 7.

[3] S. W. Hawking and G. F. R. Ellis, The Large-Scale Structure of Space-time (Cambridge University Press, Cambridge, England, 1973).

[4] R. M. Wald, General Relativity (University of Chicago Press, Chicago, 1984).

[5] P. T. Chruściel, in Differential Geometry and Mathematical Physics, edited by J. Beem and K. L. Dugal (American Mathematical Society, Providence, 1994).

[6] B. Carter, in Black Holes, edited by C. DeWitt and B. S. DeWitt (Gordon and Breach Science Publishers, New York, 1973), pp. 57-214.

[7] R. Penrose, Rev. Nuovo Cimento, 1, 252 (1969).

[8] R. Penrose, in Theoretical Principles in Astrophysics and Relativity, edited by N. R. Lebovitz, W. H. Reid, and P. O. Vandervoort (University of Chicago Press, 1978), Chap. 9.

[9] R. Penrose, in General Relativity: An Einstein Centenary Survey, edited by S. W. Hawking and W. Israel (Cambridge University Press, Cambridge, England, 1979), Chap. 12.

[10] R. P. Geroch and G. T. Horowitz, in General Relativity: An Einstein Centenary Survey, edited by S. W. Hawking and W. Israel (Cambridge University Press, Cambridge, England, 1979). Chap. 5.

[11] R. Penrose, Phys. Rev. Lett. 14, 57 (1965).

[12] E. Poisson and W. Israel, Phys. Rev. D 41, 1796 (1990).

[13] C. Barrabès, W. Israel, and E. Poisson, Class. Quantum Grav. 7, L273 (1990).

[14] A. Bonanno, S. Droz, W. Israel, and S. M. Morsink, Phys. Rev. D 50, 7372 (1994).

[15] A. Bonanno, S. Droz, W. Israel, and S. M. Morsink, University of Alberta Report No. Alberta-Thy-35-94 grqc/9411050).

[16] A null infinity associated with an asymptotically flat spacetime is denoted here by $\mathcal{J}$, which is the disjoint union of a past part $\mathcal{J}^{-}$(past null-infinity) and a future part $\mathcal{J}^{+}$(future null-infinity). Spatial infinity is denoted by $i^{0}$. (See Ref. for a discussion of these notions.)

[17] J. L. Friedman, K. Schleich, and D. M. Witt, Phys. Rev. Lett. 71, 1486 (1993).

[18] A spacetime with Ricci tensor $R_{a b}$ and Einstein tensor $G_{a b}$ is said to satisfy: (1) the null-convergence condition (also known as the null energy condition) if $R_{a b} k^{a} k^{b} \geq 0$ (equivalently $G_{a b} k^{a} k^{b} \geq 0$ ) for all null $k^{a}$; (2) the timelike-convergence condition (also known as the strong energy condition) if $R_{a b} t^{a} t^{b} \geq 0$ for all timelike $t^{a}$; (3) the non-negative-energy condition (also known as the weak energy condition) if $G_{a b} t^{a} t^{b} \geq 0$ for all timelike $t^{a}$; (4) the dominant-energy condition if $G_{a b} t^{a} u^{b} \geq 0$ for all future-directed timelike $t^{a}$ and $u^{b}$; (5) the non-negativepressures condition if $G_{a b} x^{a} x^{b} \geq 0$ for all spacelike $x^{a}$.

[19] S. A. Hayward, Phys. Rev. D 49, 831 (1994).

[20] G. W. Gibbons, Commun. Math. Phys. 27, 87 (1972).

[21] R. Penrose, Ann. NY Acad. Sci. 224, 125 (1973).

[22] P. S. Jang and R. W. Wald, J. Math. Phys. 18, 41 (1977). 
[23] P. S. Jang, Phys. Rev. D 20, 834 (1979).

[24] G. W. Gibbons, in Global Riemannian Geometry, edited by T. J. Willmore and N. J. Hitchin (Ellis Horwood, Chichester, England, 1984), pp. 194-202.

[25] G. A. Burnett, Phys. Rev. D 43, 1143 (1991).

[26] G. A. Burnett, Phys. Rev. D 48, 5688 (1993).

[27] G. A. Burnett, Phys. Rev. D 50, 6158 (1994).

[28] G. A. Burnett, Phys. Rev. D 51, 1621 (1995).

[29] J. D. Barrow, G. J. Galloway, and F. J. Tipler, Mon. Not. R. Astron. Soc. 223, 835 (1985).

[30] J. D. Barrow and F. J. Tipler, Mon. Not. R. Astron. Soc. 216, 395 (1985).

[31] J. D. Barrow and F. J. Tipler, The Anthropic Cosmological Principle (Oxford University Press, Oxford, England, 1988).

[32] J. E. Marsden and F. J. Tipler, Phys. Rep. 66, 109 (1980).

[33] J. R. Oppenheimer and H. Snyder, Phys. Rev. 56, 455 (1939).

[34] C. W. Misner, K. S. Thorne, and J. A. Wheeler, Gravitation (W. H. Freeman and Company, San Francisco, 1973).

[35] D. R. Brill, in Proceedings of the Third Marcel Grossmann Meeting on the Recent Developments of General Relativity, edited by H. Ning (North Holland, New York, 1983).

[36] R. Bartnik, Commun. Math. Phys. 117, 615 (1988).

[37] R. Bartnik, in Proceedings of the Fifth Marcel Grossmann Meeting on General Relativity, edited by D. G. Blair and M. J. Buckingham (World Scientific, Singapore, 1989).

[38] M. H. Protter and H. F. Weinberger, Maximum Principles in Differential Equations (Springer-Verlag, New York, 1984).

[39] R. Bartnik, Commun. Math. Phys. 94, 155 (1984).

[40] R. Bartnik, P. T. Chruściel, and N. Ó Murchadha, Commun. Math. Phys. 130, 95 (1990).

[41] D. M. Witt, Phys. Rev. Lett. 57, 1386 (1986).

[42] D. M. Witt, (unpublished).

[43] M. Gromov and H. B. Lawson, Jr., Inst. Hautes Etudes Sci. Publ. Math. 58, 83 (1983).

[44] R. P. Geroch, J. Math. Phys. 23, 611 (1982). 